\title{
Core-collapse supernovae in low-metallicity environments and future all-sky transient surveys
}

\author{
D. R. Young ${ }^{1}$, S. J. Smartt ${ }^{1}$, S. Mattila ${ }^{1,2}$, N. R. Tanvir ${ }^{3}$, D. Bersier ${ }^{4}$, K. C. Chambers ${ }^{5}$, N. Kaiser ${ }^{5}$, and J. L. Tonry ${ }^{5}$ \\ 1 Astrophysics Research Centre, School of Maths and Physics, Queen's University Belfast, Belfast BT7 1NN, Northern Ireland, UK \\ e-mail: dyoung06@qub.ac.uk \\ 2 Tuorla Observatory, University of Turku, Väisäläntie 20, 21500 Piikkiö, Finland \\ 3 Department of Physics and Astronomy, University of Leicester, Leicester, LE1 7RH, UK \\ 4 Astrophysics Research Institute, Liverpool John Moores University, Twelve Quays House, Egerton Wharf, Birkenhead, CH41 1LD, \\ UK \\ 5 Institute for Astronomy, University of Hawaii, 2680 Woodlawn Drive, Honolulu, HI 96822
}

Received 12 September 2007 / Accepted 17 July 2008

\section{ABSTRACT}

\begin{abstract}
Aims. Massive stars in low-metallicity environments may produce exotic explosions such as long-duration gamma-ray bursts and pair-instability supernovae when they die as core-collapse supernovae (CCSNe). Such events are predicted to be relatively common in the early Universe during the first episodes of star-formation. To understand these distant explosions it is vital to study nearby $\mathrm{CCSNe}$ arising in low-metallicity environments to determine if the explosions have different characteristics to those studied locally in high-metallicity galaxies. Many of the nearby supernova searches concentrate their efforts on high star-formation rate galaxies, hence biasing the discoveries to metal rich regimes. Here we determine the feasibility of searching for these CCSNe in metal-poor dwarf galaxies using various survey strategies.

Methods. We determine oxygen abundances and star-formation rates for all spectroscopically typed star-forming galaxies in the Sloan Digital Sky Survey, Data Release 5, within $z=0.04$. We then estimate the CCSN rates for sub-samples of galaxies with differing upper-metallicity limits. Using Monte-Carlo simulations we then predict the fraction of these CCSNe that we can expect to detect using different survey strategies. We test survey capabilities using a single $2 \mathrm{~m}$ telescope, a network of $2 \mathrm{~m}$ telescopes, and the upcoming all-sky surveys of the Pan-STARRS and LSST systems.

Results. Using a single $2 \mathrm{~m}$ telescope (with a standard CCD camera) search we predict a detection rate of $\sim 1.3 \mathrm{CCSNe}^{-1}$ in galaxies with metallicities below $12+\log (\mathrm{O} / \mathrm{H})<8.2$ which are within a volume that will allow detailed follow-up with $4 \mathrm{~m}$ and $8 \mathrm{~m}$ telescopes $(z=0.04)$. With a network of seven $2 \mathrm{~m}$ telescopes we estimate $\sim 9.3 \mathrm{CCSNe} \mathrm{yr}^{-1}$ could be found, although this would require more than $1000 \mathrm{~h}$ of telescope time allocated to the network. Within the same radial distance, a volume-limited search in the

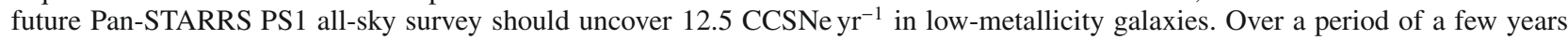
this would allow a detailed comparison of their properties. We then extend our calculations to determine the total numbers of CCSNe that can potentially be found in magnitude-limited surveys with PS1 $\left(24000 \mathrm{yr}^{-1}\right.$, within $\left.z \lesssim 0.6\right)$, PS4 (69 $000 \mathrm{yr}^{-1}$, within $\left.z \lesssim 0.8\right)$ and LSST (160000 $\mathrm{yr}^{-1}$, within $\left.z \lesssim 0.9\right)$ surveys.
\end{abstract}

Key words. surveys - stars: supernovae: general - gamma-rays: bursts

\section{Introduction}

Upon exhausting all of its available fuel, the iron core of a massive star $\left(\gtrsim 8 M_{\odot}\right)$ collapses, often giving rise to an extremely powerful, bright explosion known as a core-collapse supernova (CCSN). Under very specific circumstances these CCSNe are also thought to be associated with another very powerful and luminous event known as a long-duration gamma-ray burst (LGRB). The circumstances that permit this extraordinary partnership are still not well understood, but it seems likely that progenitors born in lower-metallicity environments (sub-solar) favour production of GRBs (see Stanek et al. 2006; Fruchter et al. 2006; Fynbo et al. 2003; Tanvir et al. 2004).

The most popular theoretical model interprets the LGRB as being caused by the core-collapse of a specific class of massive star. This fundamental theory, coined as the "Collapsar" model, was first conceived by Woosley (1993) (also see Woosley \& MacFadyen 1999). The criteria for the creation of a LGRB resulting from the collapse of a massive star are that a black-hole must be formed upon core-collapse, either promptly or via fallback of ejected material, and that an accretion disk is allowed to form around this black-hole which acts as a "central engine" causing the release of collimated relativistic jets along the rotation axis of the black-hole (Hirschi et al. 2006; Yoon et al. 2008; Tchekhovskoy et al. 2008). It is these relativistic jets that form the LGRB and the associated afterglow emission. These criteria are found to place very specific constraints on the type of progenitor star that can collapse to produce a LGRB. The core of the progenitor must be massive enough to produce a black-hole, it must be rotating rapidly enough to form an accretion disk, and the star needs to be stripped of its hydrogen envelope, so that the resulting relativistic jets are not inhibited from reaching the stellar surface. All of this points toward rapidly rotating WolfRayet stars as viable progenitors (Detmers et al. 2008; Woosley \& Heger 2006; Woosley \& Bloom 2006).

Rapidly rotating O-stars which mix-up their core processed material to produce WR stars through chemically homogeneous evolution have been suggested to satisfy the requirements of the collapsar model (Maeder 1987; Yoon \& Langer 2006; Yoon et al. 2006; Woosley \& Heger 2006). This model prevents the depletion of angular momentum due to mass-loss and allows a 
massive star to become a WR star directly, avoiding the supergiant phase. Yoon et al. (2006) have estimated that for a rapidly rotating star to be the progenitor of an LGRB, prior to collapse it must have a helium core mass greater than $10 M_{\odot}$, that is an initial mass greater than 25-30 $M_{\odot}$. Woosley \& Heger (2006) have modelled the WR mass-loss rates of Vink \& de Koter (2005) and predict that the upper metallicity limit for forming LGRB may be as high as $0.3 Z_{\odot}$. Apart from the single star progenitor model, a rapidly rotating massive star could lose its hydrogen envelope and still retain a large fraction of its initial angular momentum if a close binary companion were to strip it of the envelope rapidly enough for the progenitor to avoid spin down (Cantiello et al. 2007; Bissaldi et al. 2007; Dainotti et al. 2007).

Whatever the model for the progenitor of the LGRB, prior to collapse we require a very rapidly rotating, massive WR star. These constraints lead to the prediction that LGRBs favour a lower-metallicity environment and that CCSNe found in association with an LGRB will be classified as type Ib or type Ic, characterised by an absence of hydrogen in their spectra (Blondin \& Tonry 2007; Filippenko 2003; Hamuy 2003). To date many LGRBs have revealed "bumps" in their afterglow emission, thought to be evidence of associated SNe (see Bloom et al. 1999; Galama et al. 2000; Della Valle et al. 2003; Levan et al. 2005). A few of these associated $\mathrm{SNe}$ have been adequately spectroscopically typed, all have been found to be broad-lined type Ic SNe, helping to confirm the theory that LGRBs are the result of the collapse of hydrogen-stripped massive stars (Galama et al. 1998; Stanek et al. 2003; Hjorth et al. 2003; Zeh et al. 2004). Modjaz et al. (2008) have found that the SNe type Ic-BL associated with an LGRB are found to inhabit a sample of host galaxies of significantly lower metallicity than the host galaxies of their type Ic-BL counterparts with no associated observed LGRB. Interestingly, there has not been a $\mathrm{SN}$ type Ic-BL without an associated observed LGRB that has been found to inhabit the same low-metallicity galaxy sample that the LGRB associated $\mathrm{SNe}$ inhabit.

Fruchter et al. (2006) have found that LGRBs are preferentially found in irregular galaxies which are significantly fainter, smaller and hence presumably of lower-metallicity than typical CCSNe hosts. Sollerman et al. (2005) make a similar point, stating that the majority of LGRBs are found in extremely blue, subluminous galaxies, similar to the Blue Compact Dwarf Galaxy population. Scannapieco (2006) has speculated that at primordial metallicities stars of up to $300 M_{\odot}$ may form and if they have low mass-loss, they may end their lives still retaining much of their original mass. Heger \& Woosley (2002) predict that those stars within the mass range $140 M_{\odot}-260 M_{\odot}$ may produce pairinstability supernovae (PISNe), huge thermonuclear explosions with energies as high as $\sim 10^{53} \mathrm{erg}$. Hence understanding $\mathrm{SNe}$ in low-metallicity environments locally could offer insights into high- $z$ GRBs and early Universe explosions. An indication that these exotic PISN events may not be beyond our present day reach is the recent SN 2006gy which has been reported to have a peak luminosity three times greater than any other supernova ever recorded reaching a magnitude of -22 (Smith et al. 2006). Hypothesised as having had a Luminous Blue Variable as a progenitor with an initial mass of $\sim 150 M_{\odot}$ this has been suggested to be the first ever PISNe detected (also see Langer et al. 2007; Ofek et al. 2007; Woosley et al. 2007). The unusual SN 2006jc defines another class of peculiar low-metallicity event, occurring spatially coincident with an outburst thought to be that of an LBV just two years previously (Pastorello et al. 2007), which has also been postulated to be related to the PISN mechanism (Woosley et al. 2007).
To date there have been many surveys specifically designed to search for $\mathrm{SNe}$ and depending on the motivation of an individual survey generally one of two different survey strategies has been employed. The first of these strategies is the pointed survey strategy; here we have a fixed catalogue of galaxies which are all individually observed with a cadence of a few days and scanned for fresh SNe. These surveys included the highly successful Lick Observatory Supernova Search (LOSS) which uses the fully robotic $0.75 \mathrm{~m}$ KAIT telescope to scan a catalogue of 7500-14000 nearby galaxies ( $z \lesssim 0.04$ ), aiming to observe each individual galaxy with a cadence of 3-5 days (Filippenko et al. 2001). With the advent of relatively inexpensive CCD technology, the ability of amateur astronomers to detect nearby SNe using this pointed survey strategy has become more than substantial and a large fraction of nearby $\mathrm{SNe}$ are now being discovered by this community (Garnavich 2006). The Southern inTermediate Redshift ESO Supernova Search (STRESS) produced a catalogue of $\sim 3000(0.05<z<0.6)$ galaxies within 21 fields observed with the ESO Wide-Field-Imager and searched for $\mathrm{SNe}$ in these hosts to determine rates and their dependency on host galaxy colour and redshift evolution (Botticella et al. 2008).

The second strategy employed by SN surveys is the area survey strategy; here an area of sky is surveyed repeatedly and image subtraction used to identify transient events including SNe. The SN Legacy Survey uses the CFHT MegaCam imager to image four one-square degree fields to search for SNe type Ia with the motivation of improving the sampling of these SNe within the redshift range $0.2<z<1.0$ (Pritchet \& The SNLS Collaboration 2005; Astier et al. 2006). The equation of State SupErNova Cosmology Experiment (ESSENCE) uses the Mosaic Imager on the Blanco $4 \mathrm{~m}$ telescope to survey equatorial fields, that have preferably been observed with other widefield surveys, to discover SN type Ia within the redshift range $0.15<z<0.74$ (Miknaitis et al. 2007). The Nearby SN Factory uses ultra-wide field CCD mosaic images from the Near-Earth Asteriod Tracking (NEAT) and Palomar-Quest survey programs to perform an area survey in a bid to find SN type Ia in the redshift range $0.03<z<0.08$ (Aldering et al. 2002). The SDSS-II Supernova Survey takes repeated images of Stripe 82, a 300 square degree southern equatorial strip of the sky, driven by the ambition of identifying and measuring the lightcurves of SNe type Ia in the intermediate redshift range $0.05<z<0.35$ (Frieman et al. 2008; Sako et al. 2008). The Texas Supernova Search search (Quimby et al. 2005b; Yuan et al. 2007) is somewhat unique in that it has a small aperture telescope $(0.45 \mathrm{~m})$ but very wide field (1.85 square degrees) and is focussed on finding nearby $\mathrm{SNe}$ in wide area searches. This survey discovered SN2006gy along with many other of the brightest known SNe ever found and we shall discuss this further in Sect. 6.

The main driver of SN surveys that employ the pointed survey strategy is basically to find as many $\mathrm{SNe}$ as possible regardless of type or characteristic. To ensure that these surveys are as efficient as possible at finding CCSNe, the galaxies catalogues used by the surveys generally consist only of the most massive galaxies with the greatest star-formation rates. As a result these galaxy catalogues tend to be heavily biased towards galaxies with high metallicity. This over arching high-metallicity bias has more than likely placed us in the situation where the vast majority of CCSNe that have occurred in low-metallicity environments (such as low-luminosity, dwarf, irregular galaxies) have remained undetected. Prieto et al. (2008) have matched the SAI supernova catalogue to the SDSS-DR4 catalogue of starforming galaxies with measured metallicities and it is clear that the vast majority of the $\mathrm{SNe}$ considered have been detected in 
areas of relatively high-metallicity; SN type II occurring in host galaxies with mean metallicity $12+\log (\mathrm{O} / \mathrm{H})=8.94 \pm 0.04$ and $\mathrm{SN} \mathrm{Ib/c}$ at $9.06 \pm 0.04$.

It is inherently interesting then to search for $\mathrm{SNe}$ specifically in low-metallicty environments, or at least without biasing the search to look at only high metallicity regimes. In this paper we shall discuss several methods to search for lowmetallicity CCSN events. First we consider compiling a catalogue of nearby, low-metallicity galaxies from pre-existing catalogues (taking SDSS DR5 as our primary survey source) and using either a single $2.0 \mathrm{~m}$ telescope or a network of $2.0 \mathrm{~m}$ telescopes to perform a pointed survey of low-metallicity galaxies in the hope of detecting a few CCSNe. Secondly we consider using a future all-sky transient survey such as the Panoramic Survey Telescope and Rapid Response System (Pan-STARRS) to perform a volume-limited survey for $\mathrm{SNe}$ and estimate how many may be found in low-metallicity galaxies. A third and final method we consider is to use a future all-sky transient survey, limited only by the limiting magnitude of the survey, to search for all CCSNe including those low-metallicity events. While this paper is primarily aimed at determining numbers of low metallicity events that could be found, the latter calculation gives an estimate of the total number of CCSNe that are likely to be harvested from these upcoming surveys.

\section{Creating galaxy catalogues for the various survey strategies}

In order to produce catalogues of galaxies that we can use for the various survey strategies to be considered, we begin with the Sloan Digital Sky Survey (SDSS) (Adelman-McCarthy \& for the SDSS Collaboration 2007). The SDSS Data Release 5 (DR5) provides photometric and spectroscopic data, within a wavelength range of 3500-9200 , for $\sim 675000$ galaxies, over an area of 5713 square degrees of the northern hemisphere out to a redshift of $z \sim 0.4$. In general we only want to detect relatively nearby CCSNe as we want to spectroscopically type and follow these $\mathrm{SNe}$ with relative ease, so to this end we introduce a distance limit of $z=0.04$ to the SDSS spectroscopic catalogue. We have used the SDSS DR5 website ${ }^{1}$ to extract the out 44041 galaxies within $z=0.04$ along with data including the petrosian magnitudes $(u, g, r, i$ and $z)$, the galactic extinctions in each filter determined following Schlegel et al. (1998), the spectroscopic redshifts, the $r$-band fibre magnitudes and the line intensities of $\mathrm{H} \alpha, \mathrm{H} \beta$, [OIII] $\lambda 5007$ and [NII] $\lambda 6584$ for each galaxy.

Of these 44041 galaxies we extract out two separate samples of galaxies. The first of these samples is classified as the high signal-to-noise sample, containing galaxies with an SDSS defined line significance indicator "nSigma" $>7 \sigma$ for all of the four spectral lines mentioned previously (as advised by DR5). The second sample is classified as the low signal-to-noise sample, containing galaxies that have not been included in the high signal-to-noise sample but exhibit "nSigma" $>5 \sigma$ in both $\mathrm{H} \alpha$ and $\mathrm{H} \beta$. The high signal-to-noise sample contains 20632 galaxies and the low signal-to-noise sample contains 8703 galaxies.

\subsection{Removing AGN}

Eventually we aim to determine a star-formation rate (SFR) for each individual galaxy in our sample in order to then determine

${ }_{1}$ SDSS DR5 website: http: //www. sdss.org/dr5

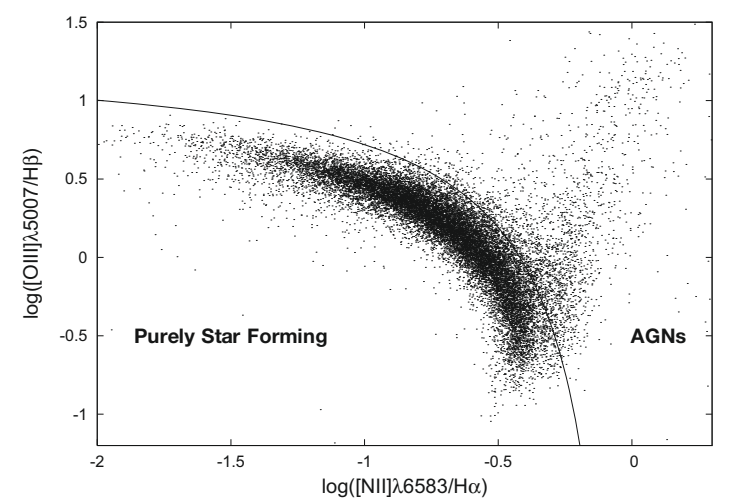

Fig. 1. [OIII] $\lambda 5007 / \mathrm{H} \beta$ vs. [NII] $\lambda 6584 / \mathrm{H} \alpha$ plot of 20632 galaxies in our SDSS DR5 good signal-to-noise galaxy sample. The diagnostic line from Kauffmann et al. (2003) discriminates between the purely star-forming galaxies in our sample (those below the line) and those hosting AGN (above the line).

their core-collapse $\mathrm{SN}$ rates (CCSR). As it is the young, massive, hot star population within each galaxy that is the dominant source of hydrogen ionising radiation, it is possible to use the $\mathrm{H} \alpha$ luminosity of these galaxies as a SFR indicator.

A difficultly arises in that many galaxies within both the high signal-to-noise and the low signal-to-noise galaxy samples will host Active Galactic Nuclei (AGN), which will also contribute to the galaxy's H $\alpha$ luminosity. To remove these AGN contaminated galaxies from the high signal-to-noise galaxy sample we use the following diagnostic line provided by Kauffmann et al. (2003) to discriminate between purely star-forming galaxies (SFGs) and galaxies that also host AGN:

$\log \left(\frac{[\mathrm{OIII}] \lambda 5007}{\mathrm{H} \beta}\right)=\frac{0.61}{\log ([\mathrm{NII}] \lambda 6584 / \mathrm{H} \alpha)-0.05}+1.3$

Fig. 1 shows the SFGs found below this line and AGN host galaxies above the line. We now have 18350 high signal-tonoise SFGs. Concerning the low signal-to-noise galaxy sample, we do not have accurate enough spectral information to apply this diagnostic line to remove any unwanted AGN host galaxies. However, following the example of Brinchmann et al. (2004) it is still possible to remove the AGN hosts from the low signalto-noise sample by requiring that $[\mathrm{NII}] \lambda 6584 / \mathrm{H} \alpha>0.6$ and that nSigma $>7 \sigma$ in both lines. We now also have 6000 low signalto-noise SFGs. An overview of all galaxy sub-samples can be found in Table 1. The 17409 unclassified galaxies are predominantly early-type galaxies that show little signs of recent starformation, which is the reason that $\mathrm{H} \alpha$ has not been detected to the significance levels that we have required. The lack of recent star-formation within these galaxies implies that they will also be void of any future CCSN events and hence these galaxies are not of interest to our survey.

Having removed all of the AGN contaminated galaxies we now have two catalogues of SFGs which we shall refer to from now on as the high signal-to-noise SFG (HSFG) catalogue and the low signal-to-noise SFG (LSFG) catalogue.

\subsection{Measuring oxygen abundances}

In order to select out low-metallicity galaxies from both the HSFG and LSFG catalogues, we define oxygen abundances using the empirical calibrations of Pettini \& Pagel (2004). Within 
Table 1. Hierarchy of galaxies extracted from the original SDSS DR5 spectroscopic galaxy sample within $z=0.04$

\begin{tabular}{rrr}
\hline \hline Sample & \multicolumn{2}{c}{ Subsample } \\
High signal-to-noise galaxies [20 632] & SFGs [18 350] & AGN [2 282] \\
Low signal-to-noise galaxies [8 703] & SFGs [6 000] & AGN [2 703] \\
Unclassified galaxies [17 409] & - & \\
\hline All SDSS DR5 Galaxies [44 041] & & - \\
\hline
\end{tabular}

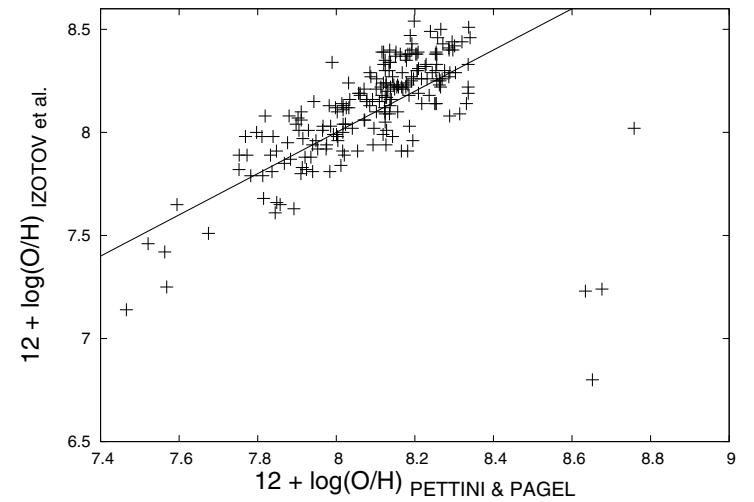

Fig. 2. Comparing our Pettini \& Pagel (2004) empirically calibrated oxygen abundances with those directly measured by Izotov et al. (2006) via measuring the electron temperature reveals that our measurements are reliable with an rms scatter of 0.14 dex, the solid line depicting a one-to-one correspondence. Note the four outlying galaxies - see text for details.

the range $8.12 \lesssim 12+\log (\mathrm{O} / \mathrm{H})<9.05$ the following empirical calibration is used:

$12+\log (\mathrm{O} / \mathrm{H})=8.73-0.32 \log \left(\frac{[\mathrm{OIII}] \lambda 5007 / \mathrm{H} \beta}{[\mathrm{NII}] \lambda 6584 / \mathrm{H} \alpha}\right)$

and below $12+\log (\mathrm{O} / \mathrm{H}) \simeq 8.12$ the following calibration is used:

$12+\log (\mathrm{O} / \mathrm{H})=8.9+0.59 \log ([\mathrm{NII}] \lambda 6584 / \mathrm{H} \alpha)$.

The wavelengths of the emission lines used for the flux ratios in both of these calibrations are separated by only a small amount and therefore their ratios are free of any extinction effects. Of the sample of 18350 HSFGs, Izotov et al. (2006) have directly measured metallicities for 209. They determined the oxygen abundances by measuring the [OIII] $\lambda 4363 /[\mathrm{OIII}] \lambda 5007$ line ratio to calculate an electron temperature $T_{\mathrm{e}}$, and then derived the abundances directly from the strengths of the [OII] $\lambda 3727$ (or [OII] $\lambda 7320,7331$ when [OII] $\lambda 3727$ was not available) and [OIII] $\lambda \lambda 4959,5007$ emission lines. Comparing the Izotov et al. $T_{\mathrm{e}}$ measured abundances with our Pettini \& Pagel empirically calibrated abundances we find good agreement, apart from four outlying galaxies (see Fig. 2). These galaxies are SDSS J124813.65-031958.2, SDSS J091731.22+415936.8, SDSS J123139.98+035631.4 and SDSS J130240.78+010426.8.

When viewed, these four outliers seem to be dwarf galaxies that are in the same line of sight as and possibly gravitationally bound to much larger and presumably more metal rich galaxies. Assuming that the contamination from these background galaxies has not been adequately removed from the spectra of the dwarf galaxies by the SDSS reduction pipeline would explain the discrepancy between the oxygen abundances measured by Izotov et al. and our measurements. Viewing a random selection of the remaining galaxies from the Izotov et al. sample reveal the blue compact dwarf galaxies expected from their low-metallicity galaxy sample. The fact that we may be overestimating the oxygen abundances of a very small fraction of galaxies should not concern us too much as we are trying to produce a low-metallicity galaxy sample and not a high-metallicity sample that would then possibly be contaminated by a few misplaced lower-metallicity galaxies. Choosing then to ignore these four outlying galaxies we find that our oxygen abundance measurements for the remaining 205 galaxies fall with an rms scatter of 0.14 dex from the directly measured abundances of the Izotov et al.

Recently Prieto et al. (2008) have taken a sample of 125958 SFGs from SDSS DR4, with oxygen abundances derived in the same fashion as Tremonti et al. (2004) used for SFGs in DR2. The Tremonti et al. (2004) method for deriving oxygen abundance estimates an individual galaxy's metallicty via a likelihood analysis which simultaneously fits multiple optical nebular emission lines to those predicted by the hybrid stellarpopulation plus photoionisation models of Charlot \& Longhetti (2001). A likelihood distribution of the metallicity is determined for each galaxy the median is taken as the best estimate of the galaxy metallicity. The Tremonti et al. (2004) metallicities are essentially on the Kewley \& Dopita (2002) abundance scale. Matching our catalogues of HSFGs and LSFGs against the sample of 125958 SFGs of Prieto et al. (2008), we find a common sample of 18014 SFGs. The oxygen abundances that we measure with the Pettini \& Pagel method are typically $\sim 0.2$ dex below that of Tremonti et al., in agreement with the findings of Modjaz et al. (2008). The cause of this discrepancy is debated but may either be due to certain parameters that produce temperature variations not being taken into consideration when deriving $T_{\mathrm{e}}$ at higher-metallicity, which would lead to an underestimation of the oxygen abundance measured on the Pettini \& Pagel scale which is calibrated with $T_{\mathrm{e}}$ measured abundances (Stasińska 2005; Bresolin 2006), or to an unknown problem with the photoionisation models used by Tremonti et al. (Kennicutt et al. 2003).

\section{3. $S N$ rate indicator}

Having measured the metallicity for each of the galaxies in our catalogues, we now wish to determine CCSRs. To do this we must first determine SFRs for the galaxies and then determine the fraction of those stars formed that will eventually end their lives as CCSNe. The best indicator that we have for the SFR for each galaxy is its $\mathrm{H} \alpha$ luminosity. As alluded to previously, it is going to be the young, massive, hot stars in purely star-forming galaxies that are the dominant source of hydrogen ionising radiation, causing the galaxy's H $\alpha$ luminosity to be proportional to its recent SFR.

Kennicutt (1998) has determined the following calibration between a galaxy's SFR and its $\mathrm{H} \alpha$ luminosity:

$\operatorname{SFR}_{\mathrm{H} \alpha}\left(M_{\odot} \mathrm{yr}^{-1}\right)=\frac{L_{\mathrm{H} \alpha}}{1.27 \times 10^{34}(\mathrm{~W})}$

where the luminosity is measured in Watts. 
Derived from their model fits, Brinchmann et al. (2004) also provide likelihood distributions for the conversion factor between the $\mathrm{H} \alpha$ luminosity and the SFR for galaxies of various mass ranges. They confirm that the Kennicutt calibration is a very good typical calibration, comparing well with the median value for their sample. When considering the complete HSFG and LSFG catalogues it is acceptable to assume a median mass range for the galaxies and we employ the Kennicutt calibration. However, when considering galaxies with relatively poor metallicity we choose to use the most probable conversion factor for the Brinchmann et al. distribution with the lowest mass range $\left(\log M_{*}<8\right)$ as this distribution closest resembles the lowmetallicity galaxies in our catalogues i.e. low-mass, blue, dwarf, irregular galaxies.

$\operatorname{SFR}_{\mathrm{H} \alpha}\left(M_{\odot} \mathrm{yr}^{-1}\right)=\frac{L_{\mathrm{H} \alpha}}{2.01 \times 10^{34}(\mathrm{~W})}$

SDSS provides the $\mathrm{H} \alpha$ equivalent line width and also the continuum flux at the wavelength of $\mathrm{H} \alpha$, the equivalent width times the continuum flux giving the $\mathrm{H} \alpha$ flux which is then used to determine the $\mathrm{H} \alpha$ luminosity. A problem with the SDSS data is that the measured $\mathrm{H} \alpha$ flux for a galaxy is only the flux which falls within the $3^{\prime \prime}$ fibre aperture of the SDSS multi-fibre spectrograph. Typically this is only a fraction of the total galaxy flux, as the SDSS spectrograph fibre locks onto the centre of the galaxy and any flux that falls outside of the $3^{\prime \prime}$ fibre aperture is lost. Hopkins et al. (2003) have developed a very simple aperturecorrection that can be applied to the measured galaxy $\mathrm{H} \alpha$ luminosity to give an estimate of the total galaxy $\mathrm{H} \alpha$ luminosity. The aperture-correction takes account of the ratio between the petrosian photometric $r$-band galaxy magnitude and the synthetic $r$-band "fibre magnitude" determined from the galaxy spectrum. Hopkins et al. also provide an extinction correction to be used when determining the galaxy $\mathrm{H} \alpha$ SFR. The correction makes use of the Balmer decrement and assumes the standard Galactic extinction law of Cardelli et al. (1989). This gives a final aperture and extinction corrected $\mathrm{H} \alpha$ luminosity SFR indicator for our entire galaxy sample as given in Eq. (6), and for the lowmetallicity galaxy sample as given in Eq. (7), where $S_{\mathrm{H} \alpha}$ and $S_{\mathrm{H} \beta}$ are the line fluxes corrected for stellar absorption according to Hopkins et al. (2003). Having now determined a SFR for each of the galaxies in our catalogues, we can compare these rates with their oxygen abundances (Fig. 3). It is clear that in general the higher the galaxy SFR the higher the typical oxygen abundance. It can be reasoned that if the SFR of a galaxy is high, or has been high at any point in its history, there is an increased population of young, hot, massive stars which in turn leads to an increased rate of CCSNe and therefore a greater rate of enrichment of the ISM. The observed high-metallicity cutoff of the SDSS galaxies is probably due to a saturation suffered by [OIII] $\lambda 4363 T_{\mathrm{e}}$ calibrated metallicities (see Kewley \& Ellison 2008)

$$
\begin{aligned}
& \operatorname{SFR}_{\mathrm{H} \alpha}\left(M_{\odot} \mathrm{yr}^{-1}\right)= \\
& 10^{-0.4\left(r_{\text {Petro }}-r_{\text {fibre }}\right)}\left[\frac{S_{\mathrm{H} \alpha} / S_{\mathrm{H} \beta}}{2.86}\right]^{2.114} \frac{L_{\mathrm{H} \alpha}}{1.27 \times 10^{34}(\mathrm{~W})} \\
& \operatorname{SFR}_{\mathrm{H} \alpha}\left(M_{\odot} \mathrm{yr}^{-1}\right)= \\
& 10^{-0.4\left(r_{\text {Petro }}-r_{\text {fibre }}\right)}\left[\frac{S_{\mathrm{H} \alpha} / S_{\mathrm{H} \beta}}{2.86}\right]^{2.114} \frac{L_{\mathrm{H} \alpha}}{2.01 \times 10^{34}(\mathrm{~W})} .
\end{aligned}
$$

Given a SFR, it is relatively simple to convert to a CCSR by determining the fraction of a stellar population that will eventually

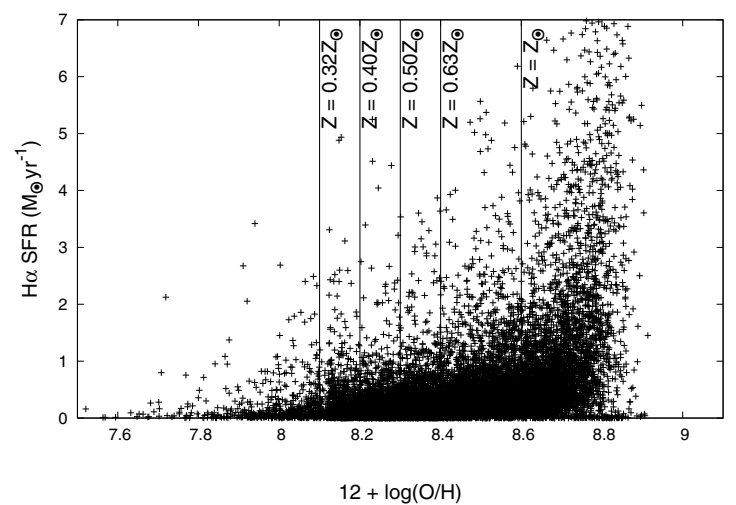

Fig. 3. $\mathrm{H} \alpha$ determined star-formation rates using the low-mass range calibration of Brinchmann et al. (2004) of 18350 galaxies in our HSFG catalogue compared with their measured oxygen abundances. In general, the greater the galaxy metallicity, the greater its star-formation rate - as expected.

collapse to create CCSNe. Following the method used by e.g. Mattila \& Meikle (2001), we use an initial mass function (IMF) for a stellar population to calculate the fraction of this population within the mass range $8 M_{\odot}<M<50 M_{\odot}$, the same mass range for stars predicted to end their lives as CCSNe. From this logic the CCSR is determined as:

$\mathrm{CCSR}=\frac{\int_{8 M_{\odot}}^{50 M_{\odot}} \phi(m) \mathrm{d} m}{\int_{0.1 M_{\odot}}^{125 M_{\odot}} m \phi(m) \mathrm{d} m} \times \mathrm{SFR}$

where $\phi(m)$ is the Salpeter IMF (Salpeter 1955) with upper and lower mass cut-offs of $0.1 M_{\odot}$ and $125 M_{\odot}$. This conversion is calculated to be:

$\operatorname{CCSR}\left(\operatorname{SNe~} \mathrm{yr}^{-1}\right)=0.007 \times \operatorname{SFR}\left(M_{\odot} \mathrm{yr}^{-1}\right)$.

\subsection{Additional nearby bright galaxies}

The target selection criteria for the SDSS DR5 spectroscopic sample includes a bright magnitude limit of $r \sim 14.5$ in order to avoid saturation and excessive cross-talk in the spectrographs. As a result of this restriction many of the nearby luminous galaxies have been omitted from the DR5 spectroscopic sample. To account for these missing bright galaxies and construct a complete galaxy catalogue we initially select out the galaxies from the HyperLeda galaxy catalogue that match with galaxies from the SDSS DR5 photometric survey with magnitudes $r<14.5$, assuming that the HyperLeda catalogue contains all of the nearby luminous galaxies. From this catalogue of matched galaxies we further select out those galaxies that are not found in the SDSS DR5 spectroscopic survey. We discover a total of 1887 nearby luminous galaxies included in the SDSS DR5 photometric survey that have been omited from the spectroscopic survey.

Of these 1887 galaxies we wish to know the fraction that are star-forming galaxies. HyperLeda provides a galaxy morphological classification for a large number of the galaxies in the catalogue and we are able to remove all galaxies with an earlytype classification, as these galaxies will generally have very low SFRs. But note that some early-type galaxies have HII regions and some evidence of low-level star-formation (e.g. Moss $\&$ Whittle 2005). The mean $g-i$ colour of these removed earlytype galaxies is 1.216 , with a standard deviation $\sigma=0.153$. In an 
Table 2. The table shows the expected CCSN rates within the SDSS DR5 spectroscopic survey area (14\% of the entire sky) out to a distance of $z \sim 0.04$.

\begin{tabular}{|c|c|c|c|c|c|c|}
\hline \multicolumn{7}{|c|}{ " HSFG catalogue } \\
\hline & \multicolumn{6}{|c|}{ Individual galaxy sn rate limits } \\
\hline & \multicolumn{2}{|c|}{$>0.0 \mathrm{SNe}_{\mathbf{y r}}^{-1}$} & \multicolumn{2}{|c|}{$>0.001{\mathrm{SNe} \mathrm{yr}^{-1}}^{-1}$} & \multicolumn{2}{|c|}{$>0.01 \mathrm{SNe}_{\mathrm{yr}^{-1}}$} \\
\hline $12+\log (\mathrm{O} / \mathrm{H})$ & Galaxies & SNe $\mathbf{y r}^{-1}$ & Galaxies & SNe $\mathrm{yr}^{-1}$ & Galaxies & SNe $\mathbf{y r}^{-1}$ \\
\hline No Limit & 18350 & 115.6 & 13974 & 113.4 & 2557 & 73.6 \\
\hline$<8.4$ & 8019 & 13.2 & 3650 & 11.2 & 120 & 2.3 \\
\hline$<8.3$ & 4290 & 6.9 & 1830 & 5.9 & 73 & 1.3 \\
\hline$<8.2$ & 1713 & 3.1 & 727 & 2.8 & 42 & 0.8 \\
\hline$<8.1$ & 537 & 1.0 & 209 & 0.9 & 16 & 0.3 \\
\hline \multicolumn{7}{|c|}{ LSFG catalogue } \\
\hline & \multicolumn{6}{|c|}{ Individual galaxy sn rate limits } \\
\hline & \multicolumn{2}{|c|}{$>0.0 \mathrm{SNe} \mathbf{y r}^{-1}$} & \multicolumn{2}{|c|}{$>0.001 \mathrm{SNe}^{-1}$} & \multicolumn{2}{|c|}{$>0.01 \mathrm{SNe}_{\mathrm{yr}}^{-1}$} \\
\hline $12+\log (\mathrm{O} / \mathrm{H})$ & Galaxies & SNe $\mathbf{y r}^{-1}$ & Galaxies & SNe $\mathbf{y r}^{-1}$ & Galaxies & SNe $\mathbf{y r}^{-1}$ \\
\hline No Limit & 6000 & 34.1 & 3691 & 33.2 & 901 & 22.9 \\
\hline$<8.4$ & 1757 & 0.8 & 116 & 0.3 & 6 & 0.1 \\
\hline$<8.3$ & 1025 & 0.3 & 50 & 0.1 & 0 & 0.0 \\
\hline$<8.2$ & 401 & 0.1 & 18 & 0.0 & 0 & 0.0 \\
\hline$<8.1$ & 116 & 0.0 & 4 & 0.0 & 0 & 0.0 \\
\hline \multicolumn{7}{|c|}{ Nearby bright galaxy catalogue } \\
\hline & & Galaxies & & SNe $\mathrm{yr}^{-1}$ & & \\
\hline & & 1216 & & 12.70 & & \\
\hline
\end{tabular}

attempt to remove the remaining non-starforming galaxies from this bright galaxy sample that do not have a morphological classification, we remove all galaxies redward of one $\sigma$ from the mean $g-i$ colour, i.e. $g-i>1.063$. This results in 1216 remaining star-forming galaxies. There is a possibility that this cut may also exclude a few starburst galaxies which have been heavily reddened due to the effects of extinction, but we are mainly concerned about low-metallicity galaxies, which are not greatly affected by extinction.

As we have no spectral information for these galaxies from SDSS DR5, it is necessary to use an alternative indicator to estimate SFR other than the $\mathrm{H} \alpha$ luminosity. The $U$-band luminosity can be used as a suitable indicator as developed by Moustakas et al. (2006):

$\operatorname{SFR}_{U_{\mathrm{obs}}}\left(M_{\odot} \mathrm{yr}^{-1}\right)=(1.4 \pm 1.1) \times 10^{-43} \frac{L_{U_{\mathrm{obs}}}}{\operatorname{ergs~s}^{-1}}$,

where the SDSS $u$-band is transformed to $U_{\text {vega }}$ using the following transformation from Blanton \& Roweis (2007):

$U_{\text {vega }}=u-0.0140(u-g)+0.0556$,

and the distance moduli from HyperLeda are used to determine $L_{U_{\text {obs }}}$. The CCSR is then determined using Eq. (9). Note that as Moustakas et al. have empirically calibrated this $U$-band SFR indicator from extinction-corrected $\mathrm{H} \alpha$ galaxy luminosities, there is no need to further correct this indicator for dust reddening. The 1216 bright, nearby galaxies have a resulting $U$-band

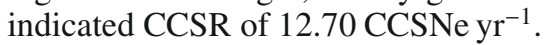

We now have three galaxy catalogues; a HSFG catalogue and a LSFG catalogue, with measured metallcities and CCSRs, and a nearby galaxy catalogue containing bright galaxies not found in the SDSS spectroscopic galaxy catalogue. We have estimates of SFRs and CCSRs for all these galaxies. Taking our catalogues of 18350 HSFGs and 6000 LSFGs and introducing various upper limits on the individual galaxy metallicities and lower limits on the CCSRs we are able to extract out separate galaxy sub-samples. Table 2 displays these sub-samples, differing both in the number of galaxies they contain and their estimated CCSR determined from the galaxy SFRs derived from aperture and extinction-corrected $\mathrm{H} \alpha$ luminosities for the HSFG and LSFG catalogues, and from $U$-band luminosities for the nearby bright galaxy catalogue. For the samples of galaxies with no metallicity constraint the SFRs have been derived using the "typical" calibration of Kennicutt (1998) whereas the SFRs of the sub-samples of galaxies with constraints on metallicity have been derived using the lowest mass range calibration of Brinchmann et al. (2004). These full catalogues or sub-samples of these catalogue can now be used to determine the feasibility of searching for low-metallicity CCSN events using various survey strategies. Figure 4 shows the cumulative distribution of the combined CCSN rate from our HSFG and LSFG catalogues, measured against oxygen abundance. It is clear that the CCSR increases steeply with oxygen abundance of the galaxy sample.

\section{Strategy 1: a pointed survey of catalogued low-metallicity galaxies}

When determining the feasibility of using a pointed lowmetallicity survey to search for CCSNe in a catalogue of lowmetallicity galaxies we consider using the fully robotic $2.0 \mathrm{~m}$ Liverpool Telescope situated at the Observatorio del Roque de los Muchachos, La Palma. We shall also consider the using of a network of similar sized telescopes.

There are three characteristics that we require of the galaxy catalogue that we shall use with this survey strategy; firstly, it must contain only a few hundred galaxies as too many galaxies in the catalogue would hinder our ability to observe each individual galaxy frequently enough to ensure that we detect any SN that it may host. Secondly, we require that the galaxies are of sufficiently low metallicity in order to determine how the CCSNe that they host differ from those hosted by their higher metallicity counterparts. Finally, the galaxies must exhibit a suitably high enough CCSR to increase the probability of detecting these 


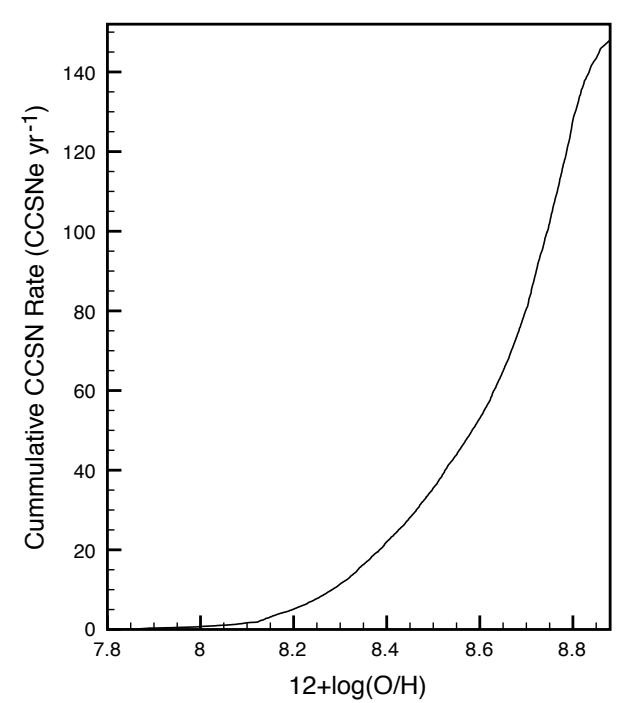

Fig. 4. Cumulative distribution of the combined CCSN rate from our HSFG and LSFG catalogues, measured against oxygen abundance. It is clear that as oxygen abundance increases so to does the rate of CCSNe.

CCSNe. The latter two requirements somewhat contradict each other because metallicity tends to scale with SFR in SFGs and therefore requiring a low-metallicity galaxy sample implies that we require galaxies with lower SFRs, and hence lower CCSRs. It is therefore essential that we produce a galaxy catalogue that can act as a compromise between these two conflicting requirements.

For the purpose of this survey strategy we decide that the galaxy catalogue that optimally fits our requirements is the subsample of the HSFG cataglogue that contain galaxies with oxygen abundances of less than 8.2 dex and a CCSR greater than 1 CCSNe every 1000 years. This low-metallicity catalogue contains 727 galaxies, a suitable number for a pointed survey, with

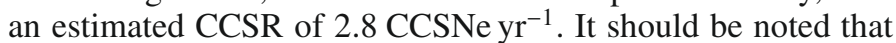
this galaxy catalogue is extracted solely from the 5713 square degrees of the sky that SDSS DR5 spectroscopic survey covers, that is $14 \%$ of the entire sky. It can be assumed that the rest of the sky contains similar density of these low-metallicity galaxies. We shall return to this point later.

\subsection{Monte-Carlo simulations}

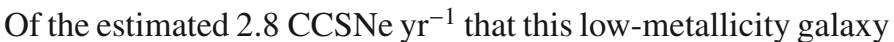
catalogue will produce, we will only be able to detect a fraction of these due to the practical limiting factors of a pointed survey. The reason that a given $\mathrm{SN}$ would not be detected is simply due to its faintness at the point of observation. The factors that will influence the likelihood that a SN will be detected when observed within our search are: whether or not the galaxy that hosts the CCSN is observable during the period of time when the CCSN is detectable, the type of CCSN (IIP, IIL, Ib, Ic or IIn) observed and its intrinsic brightness, the distance to the host galaxy, the extinction towards the CCSN, the exposure time and the age of the CCSN when observed (affected by the cadence of the observations).

By running a Monte-Carlo simulation, constrained by each of these parameters, to randomly produce a sample of 100000 possible SNe observable within our search, we can infer the fraction of CCSNe that we should actually detect.
Table 3. Relative CCSRs taken from Smartt et al. (2007).

\begin{tabular}{cccc}
\hline \hline SN type & Number & Relative rate & Core-collapse only \\
\hline Ia & 25 & $24.8 \%$ & - \\
IIP & 43 & $42.6 \%$ & $56.6 \%$ \\
IIL & 2.5 & $2.4 \%$ & $3.3 \%$ \\
Ib/c & 28 & $27.7 \%$ & $36.6 \%$ \\
IIn/P & 1.25 & $1.2 \%$ & $1.6 \%$ \\
IIn/L & 1.25 & $1.2 \%$ & $1.6 \%$ \\
\hline
\end{tabular}

\subsection{Supernova rates, template lightcurves and distributions}

In order for the Monte-Carlo simulation (MCS) to accurately reproduce the relative rates of the different types of CCSNe, we use the observed rates compiled by Smartt et al. (200), given in Table 3 . These rates have been compiled within a time and volume-limited sample, accounting for all SNe discovered within the eight year period between 1999 January 1 and 2006 December 2006 in galaxies with a recessional velocity $<2000 \mathrm{~km} \mathrm{~s}^{-1}$. Apart from those SNe that inhabit environments of heavy extinction or first observed late into their evolution, it is expected that within this appointed distance limit $(\mu=32.3)$ all known types of SNe should have been bright enough to have been detected, implying that these relative rates are as free from any Malmquist bias as possible. CCSNe of type IIb have been merged with type Ib/c, and type IIn have been divided between those with a plateau phase in the tail of their lightcurves, IIn/P, and those with a linear phase, IIn/L.

We also supply template lightcurves of the various $\mathrm{SNe}$ for the MCS. For type IIP we use 1999em as our template, taking the data from Hamuy et al. (2001). For type IIL we use 1998S, taking data for the rise to maximum light from Liu et al. (2000) and data for the tail from Fassia et al. (2000). For type Ib/c we use 2002ap, taking data from Foley et al. (2003). For SNe of type IIn, we suggest that it is appropriate to divide the relative rate evenly between type IIn that exhibit a plateau phase in their lightcurves and those that exhibit a linear phase, type IIn/P and type IIn/L respectively. For the type IIn/P, we use $1994 \mathrm{Y}$ as the template taking data from Ho et al. (2001), allowing 1998S to provide the rise to maximum light. For type IIn/L we use 1999el as the template taking data from Di Carlo et al. (2002), again allowing $1998 \mathrm{~S}$ to provide the rise to maximum light (see Fig. 5 for comparison). The following conversion from Blanton \& Roweis (2007) is used to transform the data for the lightcurves to the Sloan $g$-band (note magnitudes are to be in AB system):

$g=B-0.03517-0.3411(B-V)$.

The SN absolute magnitude distributions of Richardson et al. (2002) are used in the MCS to provide weighted, random distributions of peak magnitudes for the SNe (Table 4). Equation (12) is again used to transform these distributions to the $g$-band, taking a $(B-V)$ colour from the epoch of peak $g$-magnitude from our template lightcurves. $\sigma$ is the range in the peak magnitude distribution and $g$-band magnitudes are given in the AB system. When choosing a filter to perform a SN search with the Liverpool telescope the $r$-band is superior to the $g$-band because it has a greater filter throughput, the CCD detector is more responsive in the $r$-band and also $\mathrm{SNe}$ are generally brighter in the $r$-band especially later in their evolution. However, the Richardson et al. distributions of SN peak magnitudes are given in the $B$-band which we can transform to the $g$-band but not to the $r$-band. It is for this reason that we simulate a SN survey in the $g$-band and then with these results we can then hypothesise the outcome of a search in the $r$-band. 


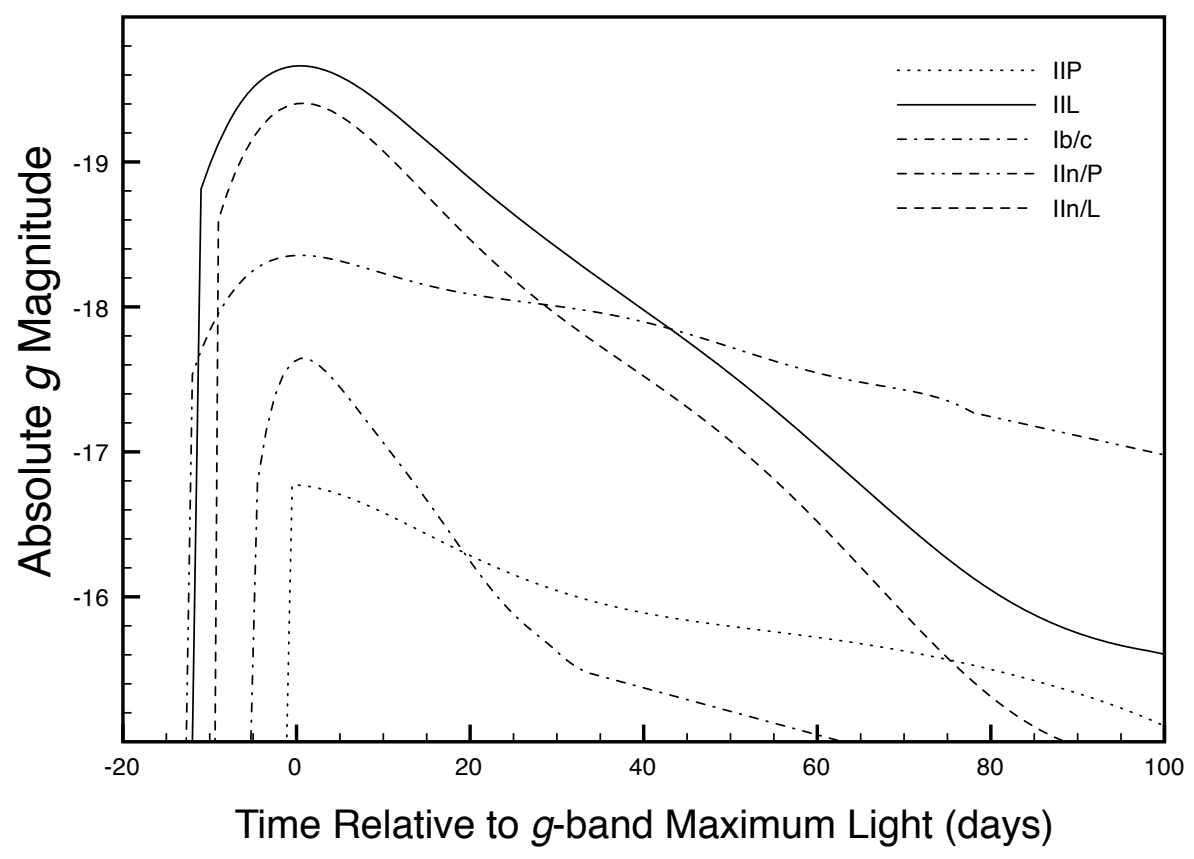

Fig. 5. Template lightcurves used within the Monte-Carlo simulation. Lightcurves for $\mathrm{SNe}$ of type IIP are according to $1999 \mathrm{em}$, type IIL to $1998 \mathrm{~S}$, type $\mathrm{Ib} / \mathrm{c}$ to $2002 \mathrm{ap}$, type $\mathrm{IIn} / \mathrm{P}$ to 1994Y and type IIn/L to 1999el. Note that all magnitudes are in the $\mathrm{AB}$ system.
Table 4. Peak magnitude distributions from Richardson et al. (2002).

\begin{tabular}{cccc}
\hline \hline SN type & $\boldsymbol{M}_{\mathrm{B}}$ & $\boldsymbol{M}_{\mathrm{g}}$ & $\sigma$ \\
\hline IIP & -17.00 & -17.02 & 1.12 \\
IIL & -18.03 & -18.00 & 0.90 \\
Ib/c & -18.04 & -18.22 & 1.39 \\
IIn/P & -19.15 & -19.24 & 0.92 \\
IIn/L & -19.15 & -19.24 & 0.92 \\
\hline
\end{tabular}

\subsection{Cadence, distance and extinction}

A major factor influencing the detection efficiency of any SN search is the time between consecutive observations of a galaxy hosting a SN. As this time increases, so does the likelihood that we will not observe the SN until it is well advanced along its lightcurve. This could result in the magnitude dropping below our detection limit, hence failing to be detected in the search. There are three parameters that affect the cadence of our search and that need to be included in the MCS. The first of these is the probability that the $\mathrm{SN}$ will be in solar conjunction for a fraction, if not all, of the time that it remains detectable by our search. To account for this factor, we use the celestial coordinates of the 727 galaxies within our low-metallicity galaxy catalogue and an almanac for the Liverpool Telescope to determine the fraction of the year that each individual galaxy is observable, producing a distribution with a mean fraction of 0.380 . We then use this distribution in the MCS to allocate a random amount of time to the cadence of observation of each SN, to account for cases where a SN would be unobservable and hence undetectable because its host galaxy is behind the sun.

The second factor that affects the cadence is the number of nights that cannot be used by the Liverpool Telescope to perform our search. Reasons why the telescope could not be used on any particular night include weather, technical difficulties and scheduled maintenance nights. To compensate for these nights in our MCS, we have taken nightly reports from the Liverpool Telescope website ${ }^{2}$, from 2005 August 1 to 2006 July 31, and use these reports to determine a distribution of nights $(58 \%$ of

${ }^{2}$ LT website: http://telescope.livjm.ac.uk/ nights in total) that can be used for our search. This distribution of usable nights is included in our MCS when considering the cadence of observations.

The final factor that will affect the cadence of observations, is the number of galaxies that we aim to observe every night. This number is influenced by the amount of telescope time dedicated to our search each night; the greater the amount of time on the telescope each night, the greater the number of galaxies we can observe per night and the higher the cadence of observations. The number of galaxies that can be observed each night is also affected by exposure time. The benefit of increasing the exposure time is that we can search to a deeper magnitude limit, meaning that fainter $\mathrm{SNe}$ are detectable and $\mathrm{SNe}$ are detectable for a greater period of time. It is essential therefore to run a number of MCSs in order to find the optimal balance between the exposure time given to each galaxy and the cadence of observations and hence enabling us to detect the greatest fraction of CCSNe hosted by our low-metallicity galaxy catalogue.

The final two parameters placed in our MCS are the host galaxy distances and the extinction toward the SNe. The MCS will attribute to each SN a random host galaxy distance, out to the distance limit of our search, which is $z=0.04$ (assuming the galaxies within our search are spread homogeneously and isotropically throughout this volume). Considering that the majority, if not all, of the galaxies within our catalogue are lowluminosity, blue, dwarf galaxies with relatively low metallicities, we assume that host galaxies will have produced very little dust and will not contribute a great deal to any extinction toward the CCSN. To this end we simply attribute a typical Galactic extinction to each $\mathrm{SN}\left(A_{\mathrm{g}}=0.3\right)$.

We note that a fraction of $\mathrm{SNe}$ remain undetected within spiral galaxies that are not observed face on (e.g. Cappellaro et al. 2003). This is due to the fact that the average extinction attributed to $\mathrm{SNe}$ in spiral galaxies with higher inclination angles is higher than that of face on spiral galaxies, making the $\mathrm{SNe}$ fainter and more difficult to detect. Implementation of a correction for this effect into the MCS, using a similar method to that of Riello \& Patat (2005), could easily result in an underestimation of the CCSRs observed in low-metallicity galaxies as these tend not be to grand-design spiral galaxies but rather 


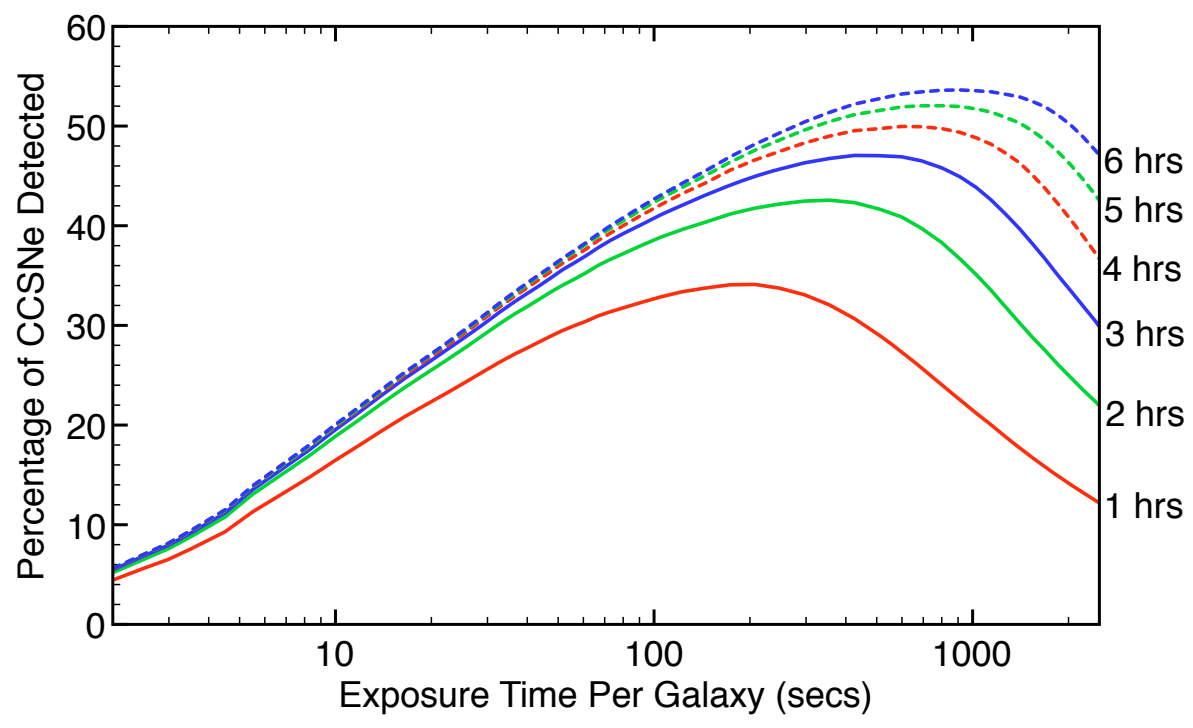

Fig. 6. Percentage of CCSNe detected from our MCSs for a differing number of hours observing per night (labeled to the right of the plot). The percentage of CCSNe detected increases as the nightly observing time increases, as this increases the cadence of observations. As the individual exposure time per galaxies increases, the limiting magnitude of the search deepens but the cadence of observations decreases. There is therefore an optimal point at which the exposure time and cadence of observation are balanced to detect the maximum percentage of SNe. dwarf, irregular galaxies where the effect of galaxy inclination would be negligible. We have therefore decided not to attempt to correct for this effect. This may make our predictions for the SN discovery rates in the full galaxy catalogues (with no metallicity limit) somewhat optimistic. However this may not lead to a significant over-estimate of events, as the SFRs that we calculate come from the observed galaxy $\mathrm{H} \alpha$ luminosities and in inclined spirals these will also be lower than in face on targets.

\subsection{Monte Carlo simulation results for a Single $2.0 \mathrm{~m}$ telescope}

Having included each of these parameters in the MCS, we randomly generate $100000 \mathrm{SNe}$ that would potentially be observable within our search. The information gained about each SN produced by the simulation include its type and its apparent magnitude at the point of observation. It is only if this magnitude is above the limiting magnitude of our search can we register the $\mathrm{SN}$ as being detected.

By running the MCS multiple times for (a) the varying number of hours we aim to observe with the LT every night and (b) the varying amount of exposure time that we dedicate to each individual galaxy, we can determine the optimal values of these parameters that shall enable us to detect the greatest fraction of the CCSNe that we have predicted our low-metallicity galaxy will produce. The results can be seen in Fig. 6.

Studying the results of the MCSs we suggest that the difference between the percentage of CCSNe detected while aiming to observe one hour per night as opposed to two hours per night or more is not enough to justify the extra observing time as the fraction of CCSN detected only increases by a few percent. As the detection rates are double valued either side of the peak detection rate, we suggest using a shorter exposure time in order to increase cadence and thereby increase the probability of discovering CCSNe earlier in their evolution as opposed to later.

Aiming to observe one hour a night using $60 \mathrm{~s}$ exposures allows each galaxy to be observed once every $\sim 17$ days while accessible with LT and enables $29.6 \%$ of CCSNe to be detected with our search. When considering the catalogue of 727 low-

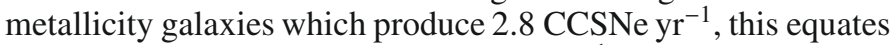
to a detected CCSN rate of $0.8 \mathrm{SNe} \mathrm{yr}^{-1}$ and requires a total amount of $154 \mathrm{~h} \mathrm{yr}^{-1}$ telescope time. Remembering that the $r$-band is superior to the $g$-band for performing a SN search, we note that given a $60 \mathrm{~s}$ exposure the $\mathrm{AB}$ limiting magnitudes for the Liverpool telescope are $g=18.7 \mathrm{mag}$ and $r=19.0 \mathrm{mag}$. Given the average cadence of observations determined by our chosen survey parameters, a typical SN will be observed $\sim 73$ days post-explosion with $g-r=0.8$ mag. Given the difference in limiting magnitudes and the typical $g-r$ colour at the point of observation, we expect to detect SNe that are 1.1 mag fainter with an $r$-band search than we would with a $g$-band search. Assuming that the template SN lightcurves and the distribution of SN peak magnitudes are similar in the $r$-band as compared to the $g$-band, we re-run the $g$-band MCS with a limiting magnitude 1.1 mag fainter than previously used. From this simulation we predict that using the $r$-band will allow for $47.8 \%$ of CCSNe to be detected with our search. Again considering the catalogue of 727 low metallicity HSFGs which

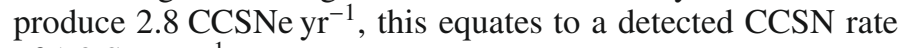
of $1.3 \mathrm{SNe} \mathrm{yr}^{-1}$.

Assuming a typical Galactic extinction of $A_{\mathrm{g}}=0.3$ $\left(A_{\mathrm{r}}=0.22\right)$ and an exposure time of $60 \mathrm{~s}$ we estimate that the absolute limiting magnitude ( $25 \sigma$ significance) for this search at a distance of $20 \mathrm{Mpc}$ will be $M_{\mathrm{r}}=-12.7$, at $70 \mathrm{Mpc}$ it will be $M_{\mathrm{r}}=-15.4$ and at $150 \mathrm{Mpc}$ it will be $M_{\mathrm{r}}=-17.1$. We plan to use the method of image matching and subtraction (see Alard \& Lupton 1998) to detect SNe throughout this survey. Assuming original images with equal depth, the process of image subtraction increases the noise by roughly a factor of $\sqrt{2}$. In addition, the detection of $\mathrm{SNe}$ inside their host galaxies will be always more difficult due to image subtraction residuals caused by uncertainties in the alignment and matching of the two images. Since we wish to avoid a large fraction of spurious detections, we require the relatively high significance level of $25 \sigma$ from the limiting magnitude of our search.

\subsection{Surveying with a network of $2.0 \mathrm{~m}$ telescopes}

As discussed previously SDSS DR5 covers only $\sim 14 \%$ of the entire sky. If we presently had the ability to compile a catalogue of low-metallicity galaxies for the whole sky as complete as that for the area of the SDSS DR5 spectroscopic survey and a collection of seven $2.0 \mathrm{~m}$ telescopes (or three to four $2.0 \mathrm{~m}$ telescopes with double the time allocation) we would expect to detect roughly 7 times the CCSNe detected solely by the LT, i.e. $\sim 9.3 \mathrm{CCSNe} / \mathrm{yr}$. We could consider using a network of six to 
eight $2.0 \mathrm{~m}$ robotic telescopes similar to the RoboNet global network of $2.0 \mathrm{~m}$ telescopes consisting of the LT and the Faulkes Telescope North and the Faulkes Telescope South to perform this kind of survey. Another advantage of using a network of telescopes in both the northern and southern hemispheres as opposed to a single telescope is that we gain a greater sky coverage and can therefore target a greater number of galaxies within our search.

The two obstacles that we would encounter if we were to use this strategy to search for CCSNe are firstly the generous amount of telescope time that we would require $\left(\sim 1000 \mathrm{~h} \mathrm{yr}^{-1}\right)$ and secondly the present lack of data required to compile an all sky galaxy catalogue. Compiling a catalogue of all galaxies listed in the $2 \mathrm{dF}, 6 \mathrm{dF}$, LEDA and SDSS DR5 within the redshift range $0<z<0.04$ results in a catalogue containing a total of 103549 individual galaxies, only a fraction of which will be star-forming. Apart from this we shall have to wait for PS1, the prototype $1.8 \mathrm{~m}$ telescope of the Panoramic Survey Telescope and Rapid Response System (Pan-STARRS), which shall cover an area of $3 \pi$ of the sky to a depth exceeding that of SDSS DR5, in order to compile a far more complete all-sky low-metallicity galaxy catalogue.

\section{Strategy 2: volume-limited searches with the Pan-STARRS all sky surveys}

Having considered a dedicated pointed low-metallicity galaxy survey to search for CCSNe both with one and with a network of $2.0 \mathrm{~m}$ telescopes, we now turn our attention to an all-sky survey. Pan-STARRS is a set of four $1.8 \mathrm{~m}$ telescopes with wide field optics, each with a 7 square degree field of view, which will be constructed by the University of Hawaii. The prototype telescope PS1 has now achieved first light and is set to go online during 2008. PS1 will have the capability of surveying the whole available sky in less than a week at a rate of 6000 square degrees per night, covering 30000 square degrees of the sky per cycle (Chambers \& Denneau 2007). Included in the list of tasks that the PS1 image reduction pipeline will perform is the subtraction of every image from a reference image in order to produce a database of residual transient objects that will include moving objects and static variables such as $\mathrm{SNe}$.

There are two different strategies that one would employ to search for $\mathrm{SNe}$ (or transients of any type), these are volumelimited searches and magnitude-limited searches. We will consider both of these. A volume-limited search has the advantage that it can quantify true rates of transients. In addition the radial limit can be chosen so that the target discoveries are bright enough to be followed in multi-wavelength studies with complementary facilities (e.g. spectroscopic and photometric follow-up with 2-8 m telescopes).

\subsection{Monte Carlo simulations}

Of all the modes in which PS1 shall be run the $3 \pi$ Steradian Survey (covering 30000 square degrees of the sky) shall be the most effective when searching for nearby CCSNe. The survey aims to cover that whole sky 60 times in 3 years, that is 12 times in each of the five filters $g, r, i, z$ and $y$. This aim has already taken historic weather patterns on Haleakala into consideration. The footprint of the survey completely covers the entire footprint of the SDSS DR5 spectroscopic survey and the AB limiting magnitude $(25 \sigma)$ in the $g$-band is stated to be 21.5 mag for a single $60 \mathrm{~s}$ exposure (Chambers \& Denneau 2007).

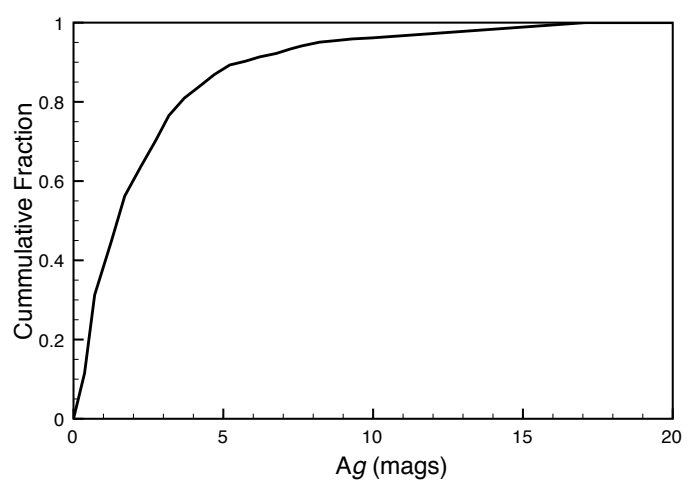

Fig. 7. Cumulative distribution of 1500 extinctions as measured through the fraction of the Galactic Plane which lies within the Pan-STARRS survey area.

Table 5. Estimated intrinsic and detectable CCSRs within the SDSS DR5 survey area and $z<0.04$. Also shown are the rates extended to the PS1 survey area.

\begin{tabular}{ccccc}
\hline \hline & \multicolumn{2}{c}{ Total sample } & \multicolumn{2}{c}{ Detected CCSNe $\mathbf{y r}^{-1}$} \\
\hline $\mathbf{1 2}+\log (\mathbf{O} / \mathbf{H})$ & Galaxies $^{-}$ & CCSNe yr $^{-1}$ & SDSS Area & PS1 Area \\
\hline No Limit & 25091 & 162.4 & 108.6 & 570.4 \\
$<\mathbf{8 . 4}$ & 9776 & 13.9 & 10.3 & 54.1 \\
$<\mathbf{8 . 3}$ & 5315 & 7.2 & 5.3 & 28.0 \\
$<\mathbf{8 . 2}$ & 2114 & 3.2 & 2.4 & 12.5 \\
$<\mathbf{8 . 1}$ & 653 & 1.1 & 0.8 & 4.3 \\
\hline
\end{tabular}

Having an adapted MCS produce 100000 potential SNe over the redshift range of our galaxy samples $(0<z<0.04)$ will again help us to estimate the fraction of potentially observable CCSNe that we will actually detect with PS1. We assume that we can take the fraction of CCSNe detected in the $g$-band as the typical fraction that would be detected in each of the five filters; indeed running the adapted MCS detailed in Sect. 5.1 in an attempt to produce the detected CCSN rates of this nearby sample in all five filters, were the $K$-corrections essentially behave as colour corrections at such low redshift, results in predicted detection efficiencies extremely close to those found if considering only the $g$-band. Concerning the cadence we have also taken the PS1 survey strategy into consideration (Chambers \& Denneau 2007), see Fig. 9. A random extinction is assigned to each of the CCSNe using a weighted distribution based on CCSRs taken from Mattila \& Meikle (2001) and Schmidt et al. (1994). However for low metallicity galaxies ( $\leq 8.2$ dex) only a typical Galactic extinction of $A_{\mathrm{g}}=0.3$ is assigned to the CCSNe, as at these metallicities we expect the galaxies to be relatively dust free.

We must also consider the fact that Pan-STARRS shall be covering a large fraction of the sky that is obscured by the Galactic Plane, a total of $\sim 7500$ square degrees of the PanSTARRS area. Any SN that is located with a line-of-sight through the Galactic Plane shall be extinguished above the typical Galactic extinction assumed. To allow for this we take the extinctions, as determined by Schlegel et al. (1998), of $\sim 1500$ points positioned homogeneously across the expanse of the Galactic Plane visible with Pan-STARRS and determine a distribution of extinctions to be folded into the MCS for any SN falling behind the Galactic Plane. The cumulative distribution of these extinctions can be seen in Fig. 7.

For the full galaxy catalogue (within $z \leq 0.04$ ) we can expect to detect $66.9 \%$ of all CCSNe. When considering the lower 
Table 6. Estimated intrinsic and detectable CCSRs within the SDSS DR5 survey area and $z<0.056$. Also shown are the rates extended to the PS4 survey area.

\begin{tabular}{ccccc}
\hline \hline & \multicolumn{2}{c}{ Total sample } & \multicolumn{2}{c}{ Detected CCSNe $\mathbf{y r}^{-1}$} \\
\hline $\mathbf{1 2}+\log (\mathbf{O} / \mathbf{H})$ & Galaxies & CCSNe $_{\mathbf{y r}}{ }^{-1}$ & SDSS area & PS4 area \\
\hline No limit & 42335 & 372.4 & 249.1 & 1308.0 \\
$<\mathbf{8 . 4}$ & 12104 & 24.6 & 18.3 & 95.8 \\
$<\mathbf{8 . 3}$ & 6053 & 11.6 & 8.6 & 45.2 \\
$<\mathbf{8 . 2}$ & 2309 & 4.7 & 3.5 & 18.3 \\
$<\mathbf{8 . 1}$ & 680 & 1.2 & 0.9 & 4.8 \\
\hline
\end{tabular}

metallicity samples we can expect to detect $74.2 \%$ of CCSNe. The predicted detectable CCSRs for both the full galaxy catalogue and for the lower-metallicity samples can be found in Table 5.

These results are a vast improvement on the rates predicted for the catalogued galaxy surveys with both a single $2.0 \mathrm{~m}$ telescope and a network of $2.0 \mathrm{~m}$ telescopes. Considering that the Pan-STARRS survey area is $\sim 5.25$ that of SDSS DR5 and given a complete galaxy catalogue of that area, we can expect to detect a total of $\sim 570 \mathrm{CCSNe} \mathrm{yr}^{-1}$, roughly 13 of which will be from host galaxies with $12+\log (\mathrm{O} / \mathrm{H})<8.2$. We would expect that the PS1 survey itself will provide a photometric catalogue of at least the quality the depth of SDSS DR5 (most likely signficcantly better) over the whole sky and hence provide us with a means to identify $\mathrm{SNe}$ in nearby, faint blue hosts. Our estimate of number of CCSNe expected to be detected in the lowmetallicity galaxy sample can be considered as a lower limit as there may be many more blue, compact, dwarf galaxies that have not been detected and catalogued by SDSS DR5. The number of CCSNe expected to be detected by PS1 in low-metallicity galaxies is hence about $50 \%$ times more than would be possible using a network of seven $2.0 \mathrm{~m}$ telescope in a dedicated pointed survey with $1000 \mathrm{~h}$ of dedicated time. It would this seem much more efficient to use the PS1 survey to discover these are objects than invest in a dedicated pointed survey with large numbers of $2.0 \mathrm{~m}$ telescopes with standard CCD cameras and fields of view.

\subsection{PS4}

Eventually three more $1.8 \mathrm{~m}$ telescopes identical to PS1 will be added to the Pan-STARRS network to create PS4. These four telescopes will be trained on the same area of the sky simultaneously to effectively perform a deeper all-sky survey (with the same observing strategy as PS1), giving a co-added $25 \sigma$ limiting magnitude of $g=22.2$.

At an apparent limiting magnitude of $m_{\mathrm{g}}=21.5$, PS1 has an absolute limiting magnitude $M_{\mathrm{g}}=-14.7$ at the boundary of our search $(z=0.04)$. For PS4 we would push the boundary of our search out to the point where the absolute limiting magnitude is identical to that of our search with PS1 in order to detect the same fraction of CCSNe as we have predicted for PS1. This distance limit is calculated to be $z=0.056$. Using the same survey strategy as previously suggested for PS1 and introducing the new limiting magnitude and distance limit, we predict that PS4 should discover a total of

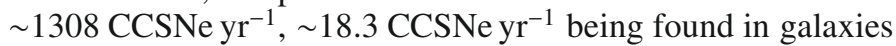
with $12+\log (\mathrm{O} / \mathrm{H})<8.2$. These results can be found in Table 6.

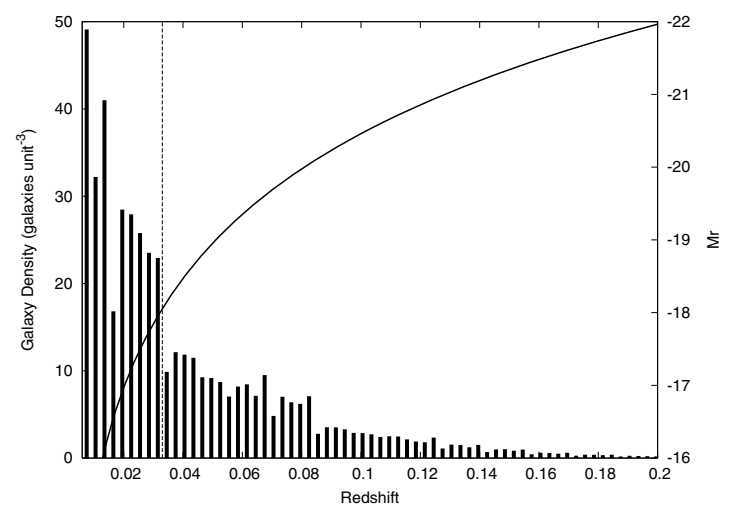

Fig. 8. The galaxy distribution for the SDSS DR5 spectroscopic survey galaxy sample. The solid curve represents the absolute magnitude limit as a function of redshift given that the SDSS DR5 apparent magnitude limit for the sample is $r=17.77$. An absolute magnitude limit of $M_{\mathrm{r}}=-18$ is reached at at redshift of 0.033 (dashed line). There is a clear drop in the galaxy density at this redshift, indicated by the dashed line, and we choose to use this limit as the completeness limit of the SDSS DR5 galaxy sample.

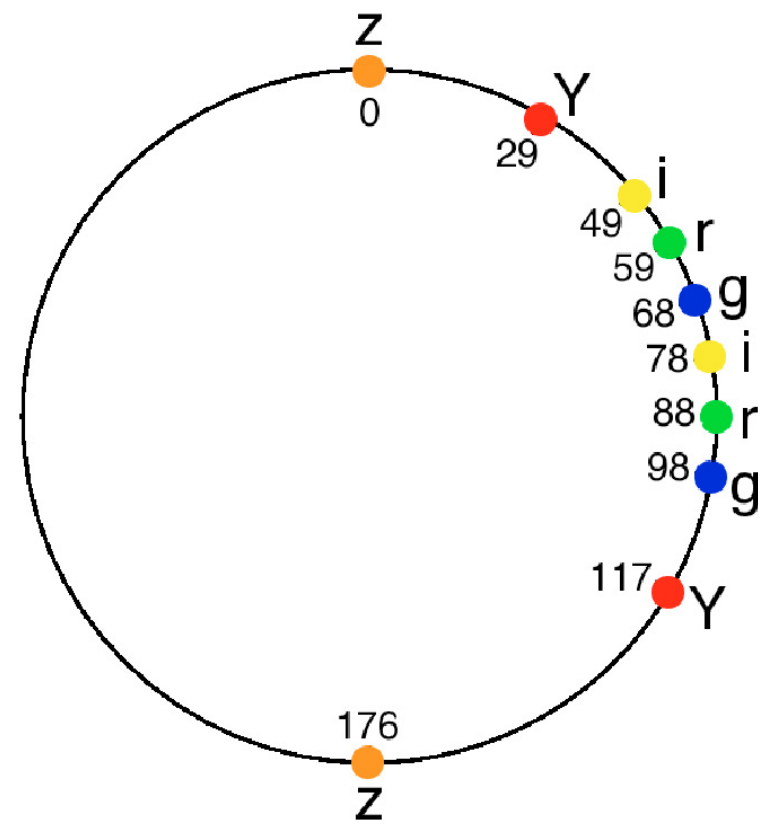

Fig. 9. A schematic depicting the cadence of observation for any given point in the sky, taken from the Pan-STARRS observation strategy (Chambers \& Denneau 2007). One Pan-STARRS year of 12 lunar months, approximately 352 days, is represented by a circle. With each observation epoch a double exposure is taken in order to identify Near Earth Objects.

\section{Strategy 3: magnitude-limited searches with future all-sky surveys}

Thus far when considering the Pan-STARRS survey to search for CCSNe we have limited ourselves to the known galaxy population within $z \lesssim 0.04$. If our aim is to detect all of the CCSNe that we possibly can then we are by no means utilizing the Pan-STARRS survey to its greatest potential. The galaxy catalogue employed is taken from SDSS DR5 and is therefore limited by a comparatively shallow depth compared to the depth to which Pan-STARRS will be able to detect CCSNe (Chambers \& Denneau 2007). 
Table 7. The $25 \sigma \mathrm{AB}$ limiting magnitudes for PS1, PS4 and LSST.

\begin{tabular}{cccc}
\hline \hline Filter & PS1 & PS4 & LSST \\
\hline$g$ & 21.5 & 22.2 & 22.7 \\
$r$ & 20.9 & 21.7 & 22.4 \\
$i$ & 20.8 & 21.6 & 21.8 \\
$z$ & 19.8 & 20.6 & 21.2 \\
$Y$ & 18.4 & 19.1 & 19.9 \\
\hline
\end{tabular}

A far more powerful method that we could implement with Pan-STARRS to detect CCSNe is to perform simply a magnitude-limited search to detect CCSNe over the whole sky and to as large distances as possible following the example of surveys such as the Nearby SN Factory (Copin et al. 2006). The inherent difficulties that we will encounter with this kind of survey will be trying to distinguish SNe from all other transient events and variables such as classical novae, asteroids and AGN and further distinguishing CCSNe from the thermonuclear (type Ia) SNe. Assuming that it may be possible to overcome these difficulties (and that we can probabilistically classify transient events) we can estimate how many CCSNe that we shall be able to detect with PS1, PS4 and also compare these rates with the likes of the Large Synoptic Survey Telescope (LSST) set to come online in 2016.

\subsection{Adapted Monte-Carlo simulations}

In order to determine the total CCSRs that we are to expect from the all-sky surveys PS1, PS4 and LSST, we must first ascertain to what redshift depth our SDSS DR5 galaxy sample is relatively complete to. When targeting galaxies for the spectroscopic survey SDSS DR5 has a lower magnitude limit of $r=17.77$. This apparent magnitude limit equates to an absolute galaxy magnitude limit of $M_{\mathrm{r}}=-18$ being reached at a redshift of $z=0.033$. The galaxy density distribution for SDSS DR5 can be seen in Fig. 8. There is a clear drop in the observed galaxy density at this redshift and we choose to set this limit as the completeness limit of the SDSS DR5 galaxy sample.

With this completeness limit in mind we can now extrapolate the complete local galaxy sample out to the observable redshift limits for the individual CCSN surveys that we are considering. We assume that the galaxy distribution throughout the observable universe is both homogeneous and isotropic. To determine the observable redshift limits of each of the suggested CCSN searches it is necessary to model the CCSN detection distribution for each of the surveys. This is made possible by using an adapted MCS to determine the percentage of CCSNe that would be detected as a function of redshift. The variant parameters included in the MCS that define one distribution from another are the cadence and the limiting magnitudes of the survey. For PS1 and PS4 we set the cadence to reflect the observing strategy for Pan-STARRS (Fig. 9) and the 25 $\sigma$ limiting magnitudes outlined by Chambers \& Denneau (2007), assuming that the limiting magnitude of PS4 is achieved via co-adding four PS1 images (see Table 7).

The current LSST configuration sets the telescope at an $8.4 \mathrm{~m}$ diameter with a 9.6 square degree field-of-view. It is planned that LSST will survey the whole of the observable sky every 3 nights, eventually covering a total of 20000 square degrees. The primary focus of the survey is to detect transients and in order to detect fast faint events the sky will be surveyed with duplicate pairs of $15 \mathrm{~s}$ exposures. Using the LSST exposure calculator this equates to the $25 \sigma$ limiting magnitudes per single exposure as found in Table 7.

When considering a magnitude-limited survey it is important to note that we shall detect $\mathrm{CCSNe}$ at relatively large redshifts compared to the volume-limited survey that we have previously discussed. With this increase in survey depth we now encounter parameters in our search that we have previously neglected due to fact that our searches have been relatively nearby. These parameters that now have to be considered in our MCS are all functions of redshift and hence could be neglected at low-redshift. They are the star-formation history (SFH) of the universe, the redshifting of light emitted by the CCSNe, the broadening of the CCSNe lightcurves due to time-dilation and finally the retardation of the CCSN rates again due to time-dilation.

Star-formation history: as star-formation was more prolific earlier in the Universe's history than it is at present, starformation is seen to increase as redshift increases (at least to moderate redshifts of $z \sim 2.5$ ). It is also important to note that approximately $50 \%$ of all local star formation activity is obscured, while at a redshift of $z \sim 1$ this fraction could be as high as $80 \%$ (Takeuchi et al. 2005). To incorporate the SFH of the universe into our simulations, but also taking into account that a greater fraction of this star-formation will be obscured by dust at higher redshifts, we include the far-ultraviolet (FUV) SFR evolution determined by Schiminovich et al. (2005), see Eq. (13). Compared with all other indicators of SFR, the FUV luminosity is the most inhibited by dust and therefore we would hope that by using this indicator we would only consider the star-formation that will give rise to CCSNe that are not so extinguished as to be invisible to our search.

$\dot{\rho}_{*}(z)=(1+z)^{2.5 \pm 0.7}$.

K corrections: comparing the bolometric fluxes of objects at various redshifts presents no difficulty as we are comparing the total flux emitted by the objects at all wavelengths. However, in practice we measure only a fraction of the total flux of an object, redshifted to the observed wavelength and transmitted through a given bandpass. This presents us with a challenge when we wish to simulate the total number of CCSNe that an all-sky survey will detect, as we have so far only considered their rest-frame (or emitted-frame) magnitudes but we must now consider their redshifted-frame (or observed-frame) magnitudes. To do this we need to calculate a transformation known as the $K$ correction (Oke \& Sandage 1968; Hogg et al. 2002), which transform between emitted-frame and observed-frame magnitudes:

$m_{\mathrm{R}}=M_{\mathrm{Q}}+\mathrm{DM}+K_{\mathrm{QR}}$

$$
\begin{aligned}
& K_{\mathrm{QR}}=-2.5 \log _{10}\left[\frac{1}{[1+z]}\right. \\
& \left.\times \frac{\int \lambda_{\mathrm{obs}} F_{\lambda}\left(\lambda_{\mathrm{obs}}\right) R\left(\lambda_{\mathrm{obs}}\right) \mathrm{d} \lambda_{\mathrm{obs}} \int \lambda_{\mathrm{em}} S_{\lambda}^{Q}\left(\lambda_{\mathrm{em}}\right) Q\left(\lambda_{\mathrm{em}}\right) \mathrm{d} \lambda_{\mathrm{em}}}{\int \lambda_{\mathrm{obs}} S_{\lambda}^{R}\left(\lambda_{\mathrm{obs}}\right) R\left(\lambda_{\mathrm{obs}}\right) \mathrm{d} \lambda_{\mathrm{obs}} \int \lambda_{\mathrm{em}} F_{\lambda}\left([1+z] \lambda_{\mathrm{em}}\right) Q\left(\lambda_{\mathrm{em}}\right) \mathrm{d} \lambda_{\mathrm{em}}}\right]
\end{aligned}
$$

where $m_{\mathrm{R}}$ is the apparent magnitude the the object as measured through observed-frame bandpass, $M_{\mathrm{Q}}$ is the absolute magnitude of the object as measured through the emitted-frame bandpass, 


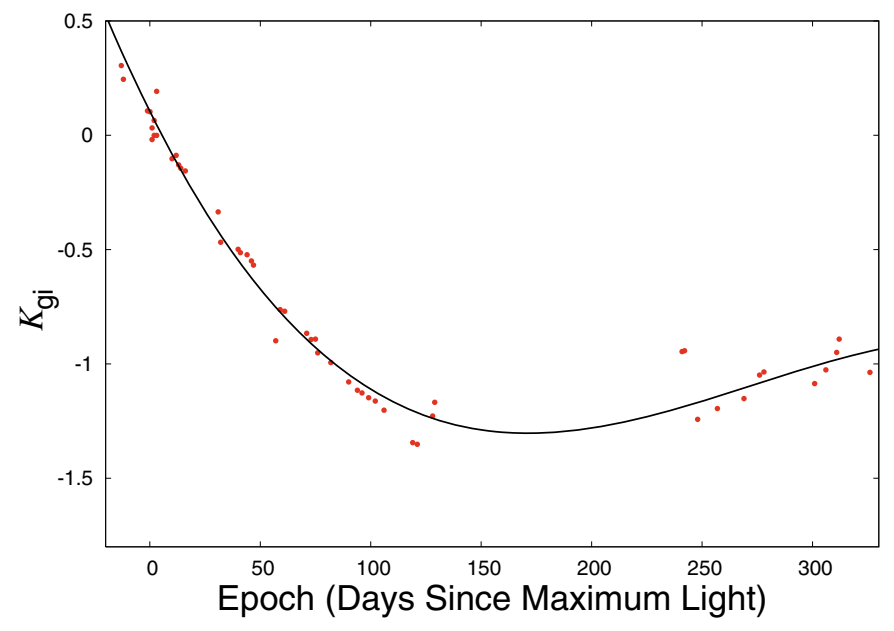

Fig. 10. A third order polynomial is fitted to each set of $K$ correction data, $K_{\mathrm{gg}}, K_{\mathrm{gr}}, K_{\mathrm{gi}}, K_{\mathrm{gz}}$ and $K_{\mathrm{gy}}$, for each CCSNe type and each redshift position sampled over the entire redshift range of the all-sky surveys considered. This example is of the $K_{\mathrm{gi}}$ correction for type IIL SNe as sampled at a redshift of $z=0.2$.

$\mathrm{DM}$ is the distance modulus of the object considered as determined from its luminosity distance and $K_{\mathrm{QR}}$ is the $K$ correction that transforms between these magnitudes. The $K$ correction can be understood as the difference in magnitude between two identical objects placed at the same distance; one traveling at a redshift of $z$ relative to the observer and measured through the observed-frame bandpass, the other at rest relative to the observer and measured through the emitted-frame bandpass.

We choose to use the $K$ correction as derived by Hogg et al. (2002) to account for the redshifting of the SN light in our MCS; see Eq. (15), where $F_{\lambda}(\lambda)$ is the spectral density of flux of the object considered taken from spectral data (measured in erg $\mathrm{cm}^{-2} \mathrm{~s}^{-1} \AA^{-1}$ ). $R(\lambda)$ and $Q(\lambda)$ are the observed-frame and emitted-frame filter total throughput functions, both depending on the various parameters of the survey considered including the total throughput of the atmosphere, the reflectivity of the telescope mirrors, the transmission of the corrector optics and filters and the quantum efficiency of the camera detector. $S_{\lambda}^{R}(\lambda)$ and $S_{\lambda}^{Q}(\lambda)$ are the spectral density of flux for the AB zeromagnitude standard source, which is a synthetic source equal to $3631 \mathrm{Jy}$ (where $1 \mathrm{Jy}=10^{-26} \mathrm{~W} \mathrm{~m}^{-2} \mathrm{~Hz}^{-1}$ ) for all frequencies (Oke \& Gunn 1983), as measured through the observed-frame and emitted-frame filters respectively.

It is essential to provide our MCS with $K$ corrections for each of the CCSNe simulated in order to determine accurate apparent magnitudes as measured through each of the five PanSTARRS filters. As the $K$ correction varies for each filter considered we must produce five $K$ corrections for each CCSN; $K_{\mathrm{gg}}, K_{\mathrm{gr}}, K_{\mathrm{gi}}, K_{\mathrm{gz}}$ and $K_{\mathrm{gy}}$. Any given CCSN produced by the simulation is placed at a random redshift and is first observed at a random point in its evolution, therefore we require $K$ corrections over the entire redshift range of our surveys, for every possible epoch of observation and for every CCSN type. It is then imperative that we have enough spectral information for each type of CCSN considered in order to cover the shear expanse of the parameters involved in calculating the $K$ corrections. We require spectral information covering a large range of the evolution of each CCSN type including the rise to maximum light and through to over 300 days post-explosion in order to accommodate for the maximum time separating two consecutive observations in the same filter according to the Pan-STARRS

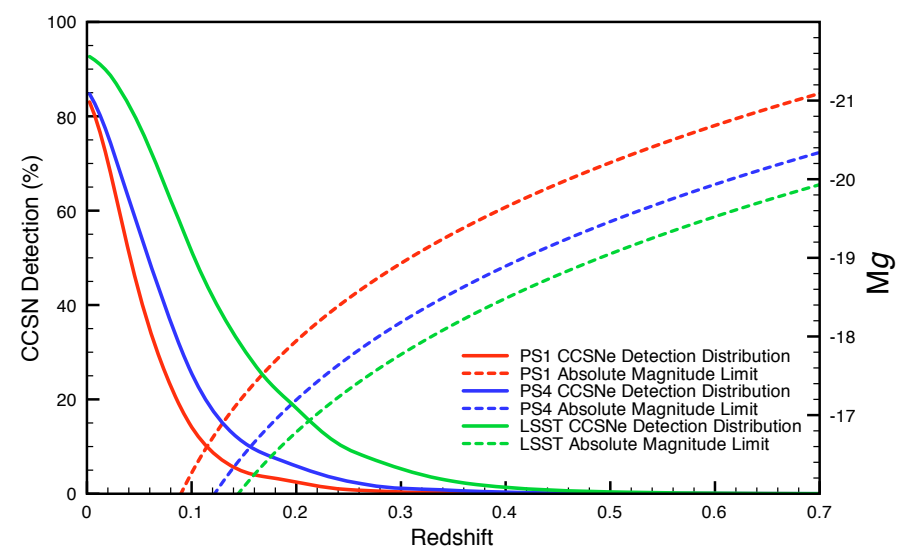

Fig. 11. The solid lines show CCSN detection distributions for PS1, PS4 and LSST, the dashed lines the absolute magnitude limits of the surveys as a function of redshift. At a absolute limiting magnitude of $M_{\mathrm{g}}=-20$ the PS1, PS4 and LSST reach their respective limiting redshifts depths of $z \sim 0.4, z \sim 0.5$ and $z \sim 0.6$.

observation strategy implemented in the MCS (see Fig. 9). As the $K$ correction evolves with time we also require spectral data for a large number of epochs in order to achieve good time resolution over the evolution of each CCSN. Finally, a wide range in wavelength coverage is required in order to provide enough information to determine $K$ corrections for all five bandpasses whenever possible, remembering that the spectral data shall be redshifted according to the distance appointed to the CCSN by the MCS.

For CCSNe of type IIP we have used the spectra of SN1999em (Hamuy et al. 2001; Leonard et al. 2001; Baron et al. 2000), for type IIL the spectra of SN1998S are used (Fassia et al. 2000; Pozzo et al. 2004; Fransson et al. 2005) and for type Ib/c the spectra of SN2002ap are used (Gal-Yam et al. 2002) supplemented with spectra from SN1990B (Matheson et al. 2001, and Asiago Catalogue) and SN1998bw (Patat et al. 2001) in order to attain the time coverage required. Due to the fact that type IIn/P and IIn/L are relatively rare, there is not enough spectral data to cover both the time and wavelength coverage required to determine a full range of $K$ corrections for these CCSNe. To compensate for this lack of data we allow the type IIn/P SN population to be represented by type IIP SNe and the type IIn/L population to be represented by type IIL SNe, adapting the Smartt et al. (2007) rates appropriately for the MCS.

Having calculated the thousands of possible $K$ corrections allowed by the relevant parameters of the MCS, we then fit a third order polynomial to each set of $K$ correction data defined by CCSN type, filter and redshift in order to provide a $K$ correction for all epochs required by the MCS (See Fig. 10)

These polynomials are read by the MCS in order to determine a final accurate apparent magnitude in each of the five filters, for each CCSN as measured at its specific redshift and point in its evolution.

Time dilation: the broadening of the CCSN lightcurves due to time dilation at relatively high redshift can be described simply as watching the CCSN evolve in "slow motion" with respect to our frame of reference. This enables us to sample the lightcurve of the CCSN with a greater frequency within its frame of reference relative to our frame of reference. The cadence hence goes as:

cadence $=$ cadence $_{\text {rest }} \times(1+z)$. 
Table 8. Predicted CCSRs for PS1, PS4 and LSST.

\begin{tabular}{ccccc}
\hline \hline \multicolumn{5}{c}{ Detected core-collapse SN rates $\left(\mathbf{S N e ~}_{\mathbf{~ y r}}{ }^{-1}\right)$} \\
\hline $\mathbf{1 2}+\log (\mathbf{O} / \mathbf{H})$ & DR5 sample $(z<0.033)$ & PS1 & PS4 & LSST \\
\hline No limit & 103.5 & 24095 & 68620 & 160249 \\
$<\mathbf{8 . 4}$ & 9.8 & 3046 & 8343 & 19627 \\
$<\mathbf{8 . 3}$ & 5.3 & 1640 & 4491 & 10566 \\
$<\mathbf{8 . 2}$ & 2.3 & 786 & 2153 & 5064 \\
$<\mathbf{8 . 1}$ & 0.9 & 278 & 762 & 1794 \\
\hline
\end{tabular}

The same argument leads to a retardation of the detected CCSN rates, giving:

$\mathrm{CCSR}=\mathrm{CCSR}_{\text {rest }} /(1+z)$.

Figure 11 shows the CCSN detection distributions for PS1, PS2 and LSST. Beyond the redshift limit where an absolute limiting magnitude of $M g=-20$ is attained the surveys will cease to detect all but the most extreme of CCSNe were luminosity is concerned and hence it is at this limit that we choose to mark the boundary of the observable universe when considering CCSNe. These redshift limits are $0.37,0.50$ and 0.59 for PS1, PS4 and LSST respectively.

Assuming that the galaxy density is homogeneous and isotropic throughout the volumes contained by these redshifts, we extrapolate our nearby local sample of galaxies and their predicted CCSRs to both the sky coverage areas and the respective redshifts for the three surveys, taking into account the SFH of the universe, the redshifting of light from distant CCSNe and the time-dilation of both the CCSN lightcurves and the CCSRs. Using the CCSN detection distributions obtained from the MCSs we then determine the detected CCSR for each of the surveys. The results can be found in Table 8 .

\section{Discussion and comparison with other surveys}

Having considered three different survey strategies designed to search for CCSNe, specifically in low-metallicity environments (a pointed survey of catalogued low-metallicity galaxies using both a single and a network of $2.0 \mathrm{~m}$ telescopes, a volume-limited survey using the Pan-STARRS all-sky surveys and finally a magnitdue limited survey using future all-sky surveys), it is now appropriate to compare these different strategies, the numbers of CCSNe we expect to detect and the various limitations of each strategy.

Using a single $2.0 \mathrm{~m}$ telescope or a network of seven $2.0 \mathrm{~m}$ telescopes to perform a pointed survey of low-metallicty galaxies within $z=0.04$ we expect to detect approxi-

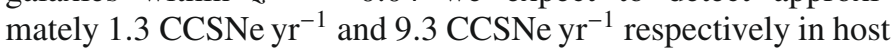
galaxies of very low-metallicity, exhibiting oxygen abundances $12+\log (\mathrm{O} / \mathrm{H})<8.2$. When considering the relative numbers of similar events that we would hope to detect using a volumelimited survey with PS1 we estimate that we would detect

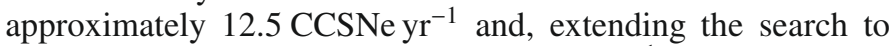

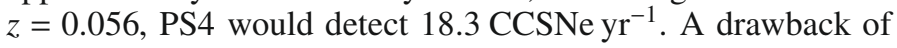
the pointed low-metallicity survey is the fact that $\sim 1000 \mathrm{~h}$ of telescope time would be required of the network of seven $2.0 \mathrm{~m}$ telescopes. Unlike the network of $2.0 \mathrm{~m}$ telescopes, the all-sky surveys have the additional advantage of not being limited by the number of galaxies that they can feasibly observe and as a result shall be capable of observing CCSN events in all environments and not only those in the lowest-metallicity host galaxies, helping us to reduce the possibility of missing any rare CCSN events of extreme interest and also gaining the capability of determining accurate relative rates of $\mathrm{SNe}$ within a large but limited vol-

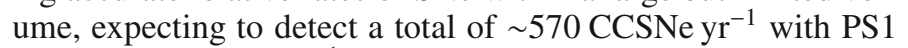
and $\sim 1300$ CCSNe $\mathrm{yr}^{-1}$ with PS4.

To estimate the amount of $8.0 \mathrm{~m}$ telescope time that we would require to spectroscopically follow the $\sim 13$ CCSNe found with the PS1 volume-limited survey in low-metallicity environments $(12+\log (\mathrm{O} / \mathrm{H})<8.2)$, we consider a CCSN occurring at the average distance of the survey, that is $\sim 120 \mathrm{Mpc}$. Depending on how early we catch this SN, we hope to observe it at various epochs during its evolution and into its tail phase. Suggesting an average absolute magnitude of -15 for these epochs equates as an observed apparent magnitude of 20.7 at $120 \mathrm{Mpc}$, including a typical galactic extinction of $0.3 \mathrm{mag}$. To determine the time required from a typical $8.0 \mathrm{~m}$ telescope for spectroscopic followup we use the VLT FORS2 exposure time calculator, selecting a suitable grism and requiring a signal-to-noise of 50 per spectral element for each spectrum. To reach this signal-to-noise level we would require a $\sim 3000 \mathrm{~s}$ on source integration. If we were to spectroscopically follow the 13 events, requiring 5 epochs of observations per SN, we would then require $\sim 70 \mathrm{~h}$ of $8.0 \mathrm{~m}$ telescope time per year.

An obstacle that would hinder both the pointed survey of low-metallicity galaxies and the volume-limited Pan-STARRS surveys is the incompleteness of any galaxy catalogue that could presently be produced. In the former case one would obviously need an all-sky target list for a pointed survey as we have demonstrated that the SDSS footprint does not have enough lowmetallicity galaxies to make a search particularly fruitful (only

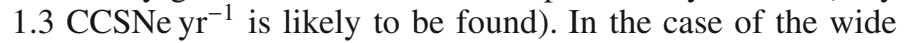
area search of Pan-STARRS one would ideally like to quantify all potential low-metallicity systems within a local volume of the Universe so that new discoveries can be quickly cross-matched and the optical transients in these galaxies immediately selected for follow-up. Hence it would be extremely useful to have an SDSS DR5 type catalogue over the entire sky. As previously discussed (see Sect. 3.5) it is currently possible to compile a catalogue of $\sim 10^{5}$ galaxies within the sky area covered by PanSTARRS and within the redshift range $z<0.04$. This catalogue is far from complete, however when PS1 completes a full cycle of sky observations we would have a deep photometric catalogue with which to search for CCSNe and classify the host galaxies.

It will be challenging to identify and select genuine CCSNe in the magnitude-limited, all sky-surveys. Without a defined volume limit it becomes impossible to describe accurate relative rates of $\mathrm{SNe}$ and without the prior knowledge of the $\mathrm{SN}$ hosts it will be very difficult to segregate CCSNe from the large numbers of other transitory events, especially more distant SNe type Ia in apparently faint (but perhaps intrinsically bright) hosts. Most of the $\mathrm{SNe}$ are likely to be too distant to have any catalogued host and one of the challenges is to classify objects from the information gathered in the all-sky surveys themselves e.g. lightcurve matching, identification and photometric redshift measurement of the host, estimated energy of the transient. If it is possible to overcome these challenges the numbers of detected CCSNe expected from these unbiased, all-sky and magnitude-limited surveys shall overwhelm those numbers from the other survey strategies far above an order of magnitude (see Table 8).

A pioneering survey in this area has been the Texas Supernova Search (TSS), which has now evolved into the ROTSE Supernova Verification Project (RSVP) (Quimby et al. 2005b; Yuan et al. 2007). The Robotic Optical Transient Search Experiment (ROTSE) presently consists of four $0.45 \mathrm{~m}$ robotic telescope located around the globe, each with a 1.85 square 

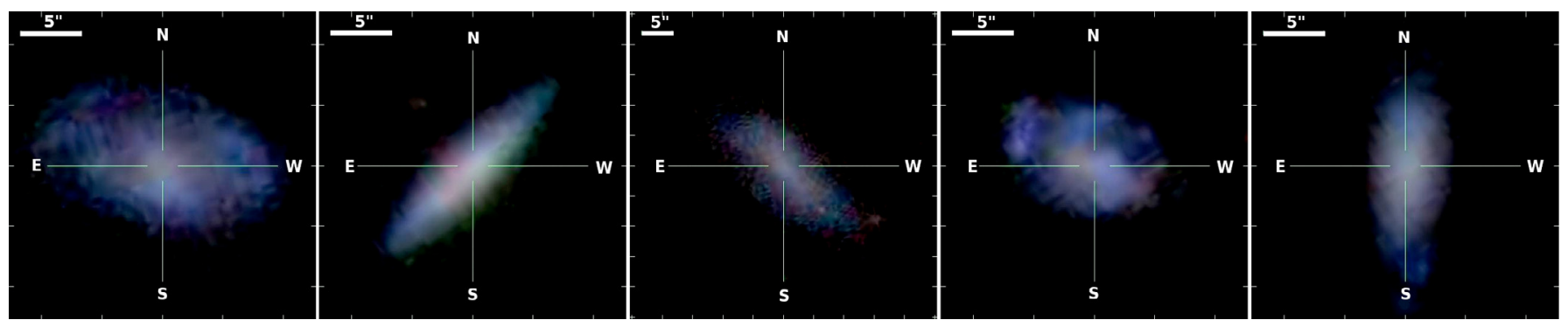

Fig. 12. Of the $12 \mathrm{SNe}$ discovered by the Nearby Supernova Factory that were hosted by SFGs in the SDSS DR5 catalogue, 5 were hosted by galaxies with $12+\log (\mathrm{O} / \mathrm{H})<8.4$. The host shown are SDSS J140737.23+384047 (SN 2007fg), SDSS J160205.11+294338 (SN 2007fz), SDSS J135042.68 + 400208 (SNF20080324-010), SDSS J154404.32+275335 (SNF20080515-004) and SDSS J150031.40+552210 (SNF20080614-002).

degree field of view, dedicated to detecting and observing optical transients, with an emphasis on GRBs. The TSS has used the ROTSE-IIIb (MacDonald Observatory Texas) to perform a wide-field search for nearby SNe. The RSVP uses all four ROTSE-III telescopes to discover SNe and other transient variables, by imaging areas of the sky that host nearby galaxy clusters. The typical cadence is 1-3 days with a typical limiting magnitude of $\sim 18.5 \mathrm{mag}$. To date the ROSTE-IIIb telescope has discovered $50 \mathrm{SNe}$ in total at mean redshift of $z=0.049$; 34 type Ia and 16 type II ( 5 of which have been classified as Ic and 5 as IIn). The most remarkable result of this small scale SN survey is that they have claim over the discovery of the three most luminous SN ever detected; SN2006tf (-20.7 mag), SN2006gy (-22 mag) and SN2005ap (-22.7 mag). SN 2006tf and SN 2006gy were both classified as a type IIn (Quimby et al. 2007a; Quimby 2006) and possibly had Luminous Blue Variable (LBV) star progenitors with explosions speculated to have been triggered by pulsation pair instability (Smith et al. 2008, 2006; Woosley et al. 2007). SN 2005ap was classified as a type II (Quimby et al. 2005a) and exhibited a broad $\mathrm{H} \alpha$ P-Cygni profile. The explosion of the progenitor, which still had its Hydrogen envelope intact, was possibly powered by either a GRB type engine or a pair-instability eruption (Quimby et al. 2007b). Although these type of super-luminous SNe may be intrinsically rare, it is more likely that they have remained undiscovered in the past due to the fact that they have been missed or misclassified as AGN in pointed surveys as they have occurred close to the cores of bright galaxies. The image-subtraction techniques used by area surveys, such as TSS and RSVP, will ensure that these types of interesting events will continue to be discovered. Taking a spectrum of the SN type IIn 1998S at peak (Fassia et al. 2000) and redshifting it to estimate the $K$-corrections that $\mathrm{SNe}$ similar to $2006 \mathrm{gy}$ would require at various redshifts, we predict that we would detect these events to $z \sim 0.6$ for PS1, $z \sim 0.8$ for PS 4 and $z \sim 0.9$ for LSST. For PS4 and LSST these limits correspond to a search volume $\sim 2.9$ and $\sim 4.4$ times greater than PS1.

The Nearby Supernova Factory (NSF) uses ultra-wide field CCD mosaic images from the Near-Earth Asteriod Tracking (NEAT) and Palomar-Quest survey programs with the aim of finding low-redshift SNe Ia (Aldering et al. 2002). Within the volume-limited $(z<0.04)$ search with PS1 we predict relativerates of SNe II, $\mathrm{SNe} \mathrm{Ib} / \mathrm{c}$ and $\mathrm{SNe}$ IIn as $57.8 \%, 38.2 \%$ and $4.0 \%$ and at mean redshifts of $0.0318,0.0316$ and 0.0321 respectively. In its lifetime the NSF had discovered and classified $85 \mathrm{CCSNe}$ within $z<0.04$ with relative-rates of $\mathrm{SNe} \mathrm{II}, \mathrm{SNe} \mathrm{Ib} / \mathrm{c}$ and SNe IIn of $67.1 \%, 20.0 \%$ and $12.9 \%$ at mean redshifts of 0.0283 , 0.0292 and 0.0284 respectively. The reason that the relative-rates of CCSNe types predicted by our PS1 volume-limited search and those discovered by the NSF are somewhat discrepant may be
Table 9. Comparison between the relative magnitudes of CCSNe we predict with a volume-limited $(z<0.04)$ search with PS1 and those discovered within the same volume by the Nearby Supernova Factory.

\begin{tabular}{ccc}
\hline \hline Magnitude range & PS1 Predictions & Classified by NSF \\
\hline$<16$ & $3.5 \%$ & $3.5 \%$ \\
$16-17$ & $6.1 \%$ & $5.9 \%$ \\
$17-18$ & $12.6 \%$ & $3.5 \%$ \\
$18-19$ & $20.3 \%$ & $47.1 \%$ \\
$19-20$ & $25.4 \%$ & $40.0 \%$ \\
$>20$ & $32.2 \%$ & $0.0 \%$ \\
\hline
\end{tabular}

due in part to the photometric screening of SN candidates that the NSF perform prior to spectroscopically classifying candidates. The NSF aim to find young SNe Ia that are nearby yet are within the Hubble-flow and therefore seem to classify SN candidates that are on the rise and that are close to the limitingmagnitude of their search $(R \sim 20 \mathrm{mag})$, as can be seen from the relative-magnitude distributions of classified SNe in Table 9. However it could be argued that because of the high-metallicity bias of many current and historic SN searches, the relativerates of nearby CCSNe compiled by Smartt et al. (200), and used in our simulations, are also biased toward higher metallicity. A high-metallicity bias in these relative-rates would be seen as an over-estimation of the true $\mathrm{SN}$ Ib/c to SN II ratio; as seen when compared to the relative-rates discovered by the NSF. Further evidence supporting this hypothesis results from cross-matching NSF discovered SNe with our catalogue of SDSS DR5 SFGs within $z<0.04$. A total of $12 \mathrm{CCSNe}$ are matched with SDSS galaxies, 5 of which occurring in galaxies with $12+\log (\mathrm{O} / \mathrm{H})<8.4$ (see Fig. 12). This high fraction of $\mathrm{CCSNe}$ discovered in low-metallicity environments may again be due to selection criteria of the NSF (possibly looking for $\mathrm{SNe}$ occurring in apparently faint hosts) but remains encouraging for our plans to search for low-metallicity CCSNe with future all-sky surveys.

Another future all-sky survey that has potential for $\mathrm{SNe}$ discoveries is Gaia: the European Space Agency's "superHipparcos" satellite with the objective of creating the most precise three-dimensional map of the Galaxy (Perryman 2005). The satellite shall have many other additional capabilities including the ability to detect nearby $\mathrm{SNe}$ (within a few hundred $\mathrm{Mpc}$ ), and predicted for launch in December 2011 it is a potential competitor of Pan-STARRS and LSST. Belokurov \& Evans (2003) have performed a feasibility study similar to this study for Gaia and

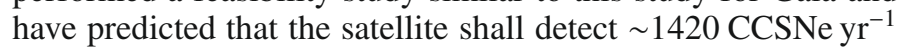
using a magnitude-limited survey strategy. Capable of detecting objects brighter than 20th magnitude, 1.5 mag brighter than the 
$g=21.5$ limit for PS1, Gaia has the ability to survey a volume of only an eighth of the depth that PS1 shall survey. Belokurov \& Evans (2003) employed a galaxy catalogue which more than likely neglected low-luminosity galaxies, which would result in the CCSN rate being under-predicted by a factor of up to $\sim 2$ fewer SNe. Hence if we scale the Belokurov \& Evans numbers by $\sim 16$, the final numbers should be comparable with our

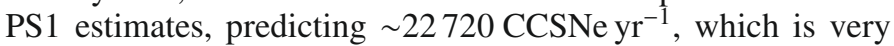
close to the rate that we have predicted for PS1 using our MCSs, $\sim 24000 \mathrm{CCSNe} \mathrm{yr}^{-1}$ (see Table 8). However, we note that these numbers are likely somewhat over optimistic due to the treatment of host galaxy extinction in our MCS.

\section{Conclusions}

Having determined oxygen abundances, star-formation rates and CCSN rates for all spectroscopically typed star-forming galaxies in the Sloan Digital Sky Survey Data Release 5 within $z=0.04$, we have used Monte-Carlo simulations to predict the fraction of these CCSNe that we can expect to detect using different survey strategies. Using a single $2 \mathrm{~m}$ telescope (with a standard CCD

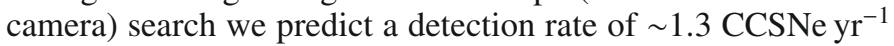
in galaxies with metallicities below $12+\log (\mathrm{O} / \mathrm{H})<8.2$ which are within a volume that will allow detailed follow-up with $4 \mathrm{~m}$ and $8 \mathrm{~m}$ telescopes $(z=0.04)$. With a network of seven

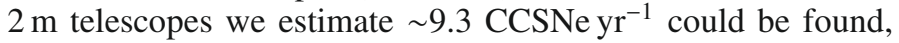
although this would require more than $1000 \mathrm{~h}$ of telescope time allocated to the network. Within the same radial distance, a volume-limited search with the future Pan-STARRS PS1 all-

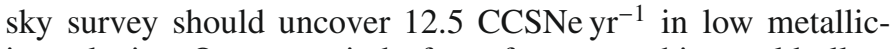
ity galaxies. Over a period of a a few years this would allow a detailed comparison of their properties. We have also extended our calculations to determine the total numbers of CCSNe that can potentially be found in magnitude-limited surveys with PS1 (24000 $\mathrm{yr}^{-1}$, within $\left.z \lesssim 0.6\right)$, PS4 (69000 $\mathrm{yr}^{-1}$, within $z \lesssim 0.8$ ) and LSST (160000 $\mathrm{yr}^{-1}$, within $\left.z \lesssim 0.9\right)$ surveys.

All considered, a final strategy chosen to searching for CCSNe in low-metallicity environments shall realistically involve both a volume-limited and a magnitude-limited all-sky survey in order to include a volume-limited galaxy sample with which to accurately determine relative $\mathrm{SN}$ rates and have some prior knowledge of the host galaxy characteristics, but yet not exclude the potential of detecting rare CCSN events that would have otherwise been missed had we only considered the volume-limited survey strategy.

With the huge number of CCSNe predicted to be detected, these all-sky surveys are set to serve as a catalyst concerning our understanding of CCSNe; including their varying characteristics with metallicity, the relative rates of the various types of $\mathrm{SNe}$ and of extremely rare events similar to SN 2006jc, SN 2006gy and more than likely events the nature of which have not yet been observed.

Acknowledgements. Funding for the Sloan Digital Sky Survey (SDSS) and SDSS-II has been provided by the Alfred P. Sloan Foundation, the Participating Institutions, the National Science Foundation, the U.S. Department of Energy, the National Aeronautics and Space Administration, the Japanese Monbukagakusho, and the Max Planck Society, and the Higher Education Funding Council for England. The SDSS Web site is http://www . sdss.org/ The SDSS is managed by the Astrophysical Research Consortium (ARC) for the Participating Institutions. The Participating Institutions are the American Museum of Natural History, Astrophysical Institute Potsdam, University of Basel, University of Cambridge, Case Western Reserve University, The University of Chicago, Drexel University, Fermilab, the Institute for Advanced Study, the Japan Participation Group, The Johns Hopkins University, the Joint Institute for Nuclear Astrophysics, the Kavli Institute for Particle Astrophysics and Cosmology, the Korean Scientist Group, the Chinese Academy of Sciences (LAMOST), Los Alamos National Laboratory, the Max-Planck-Institute for Astronomy (MPIA), the Max-Planck-Institute for Astrophysics (MPA), New Mexico State University, Ohio State University, University of Pittsburgh, University of Portsmouth, Princeton University, the United States Naval Observatory, and the University of Washington.

This research has made use of the CfA Supernova Archive, which is funded in part by the National Science Foundation through grant AST 0606772.

This work, conducted as part of the award "Understanding the lives of massive stars from birth to supernovae" (S.J. Smartt) made under the European Heads of Research Councils and European Science Foundation EURYI (European Young Investigator) Awards scheme, was supported by funds from the Participating Organisations of EURYI and the EC Sixth Framework Programme. S.J.S. and D.R.Y. thank the Leverhulme Trust and DEL for funding. S.M. acknowledges financial support from the Academy of Finland, project 8120503.

\section{References}

Adelman-McCarthy, J. K., \& for the SDSS Collaboration 2007, in press [arXiv: 0707.3380]

Alard, C., \& Lupton, R. H. 1998, ApJ, 503, 325

Aldering, G., Adam, G., Antilogus, P., et al. 2002, in Survey and Other Telescope Technologies and Discoveries, ed. J. A. Tyson, \& S. Wolff, Proc. SPIE, 4836, 61

Astier, P., Guy, J., Regnault, N., et al. 2006, A\&A, 447, 31

Baron, E., Branch, D., Hauschildt, P. H., et al. 2000, ApJ, 545, 444

Belokurov, V. A., \& Evans, N. W. 2003, MNRAS, 341, 569

Bissaldi, E., Calura, F., Matteucci, F., Longo, F., \& Barbiellini, G. 2007, in press [arXiv:astro-ph/0702652]

Blanton, M. R., \& Roweis, S. 2007, AJ, 133, 734

Blondin, S., \& Tonry, J. L. 2007, ApJ, 666, 1024

Bloom, J. S., Kulkarni, S. R., Djorgovski, S. G., et al. 1999, Nature, 401, 453

Botticella, M. T., Riello, M., Cappellaro, E., et al. 2008, A\&A, 479, 49 Bresolin, F. 2006, ArXiv Astrophysics e-prints

Brinchmann, J., Charlot, S., White, S. D. M., et al. 2004, MNRAS, 351, 1151

Cantiello, M., Yoon, S.-C., Langer, N., \& Livio, M. 2007, A\&A, 465, L29

Cappellaro, E., Barbon, R., \& Turatto, M. 2003, in press

[arXiv: astro-ph/0310859]

Cardelli, J. A., Clayton, G. C., \& Mathis, J. S. 1989, ApJ, 345, 245

Chambers, K. C., \& Denneau, L. J. 2007

Charlot, S., \& Longhetti, M. 2001, MNRAS, 323, 887

Copin, Y., Blanc, N., Bongard, S., et al. 2006, New Astron. Rev., 50, 436

Dainotti, M. G., Bernardini, M. G., Bianco, C. L., et al. 2007, A\&A, 471, L29

Della Valle, M., Malesani, D., Benetti, S., et al. 2003, A\&A, 406, L33

Detmers, R. G., Langer, N., Podsiadlowski, P., \& Izzard, R. G. 2008, ArXiv e-prints, 804

Di Carlo, E., Massi, F., Valentini, G., et al. 2002, ApJ, 573, 144

Fassia, A., Meikle, W. P. S., Vacca, W. D., et al. 2000, MNRAS, 318, 1093

Filippenko, A. V. 2003, in From Twilight to Highlight: the Physics of Supernovae, ed. W. Hillebrandt, \& B. Leibundgut, 171

Filippenko, A. V., Li, W. D., Treffers, R. R., \& Modjaz, M. 2001, in ASP Conf. Ser. 246, IAU Colloq. 183: Small Telescope Astronomy on Global Scales, ed. B. Paczynski, W.-P. Chen, \& C. Lemme, 121

Foley, R. J., Papenkova, M. S., Swift, B. J., et al. 2003, PASP, 115, 1220

Fransson, C., Challis, P. M., Chevalier, R. A., et al. 2005, ApJ, 622, 991

Frieman, J. A., Bassett, B., Becker, A., et al. 2008, AJ, 135, 338

Fruchter, A. S., Levan, A. J., Strolger, L., et al. 2006, Nature, 441, 463 Fynbo, J. P. U., Jakobsson, P., Möller, P., et al. 2003, A\&A, 406, L63 Gal-Yam, A., Ofek, E. O., \& Shemmer, O. 2002, MNRAS, 332, L73 Galama, T. J., Vreeswijk, P. M., van Paradijs, J., et al. 1998, Nature, 395, 670 Galama, T. J., Tanvir, N., Vreeswijk, P. M., et al. 2000, ApJ, 536, 185 Garnavich, P. M. 2006, Journal of the American Association of Variable Star Observers (JAAVSO), 35, 42

Hamuy, M. 2003, ArXiv Astrophysics e-prints

Hamuy, M., Pinto, P. A., Maza, J., et al. 2001, ApJ, 558, 615

Heger, A. \& Woosley, S. E. 2002, ApJ, 567, 532

Hirschi, R., Meynet, G., \& Maeder, A. 2006, ArXiv Astrophysics e-prints

Hjorth, J., Sollerman, J., Møller, P., et al. 2003, Nature, 423, 847

Ho, W. C. G., Van Dyk, S. D., Peng, C. Y., et al. 2001, PASP, 113, 1349

Hogg, D. W., Baldry, I. K., Blanton, M. R., \& Eisenstein, D. J. 2002, in press [arXiv: astro-ph/0210394]

Hopkins, A. M., Miller, C. J., Nichol, R. C., et al. 2003, ApJ, 599, 971

Izotov, Y. I., Stasińska, G., Meynet, G., Guseva, N. G., \& Thuan, T. X. 2006, A\&A, 448, 955

Kauffmann, G., Heckman, T. M., Tremonti, C., et al. 2003, MNRAS, 346, 1055 Kennicutt, Jr., R. C. 1998, ARA\&A, 36, 189

Kennicutt, Jr., R. C., Bresolin, F., \& Garnett, D. R. 2003, ApJ, 591, 801 
Kewley, L. J., \& Dopita, M. A. 2002, ApJS, 142, 35

Kewley, L. J., \& Ellison, S. L. 2008, ArXiv e-prints, 801

Langer, N., Norman, C. A., de Koter, A., et al. 2007, in press [arXiv:0708.1970]

Leonard, D. C., Filippenko, A. V., Ardila, D. R., \& Brotherton, M. S. 2001, ApJ, 553,861

Levan, A., Nugent, P., Fruchter, A., et al. 2005, ApJ, 624, 880

Liu, Q.-Z., Hu, J.-Y., Hang, H.-R., et al. 2000, A\&AS, 144, 219

Maeder, A. 1987, A\&A, 178, 159

Matheson, T., Filippenko, A. V., Li, W., Leonard, D. C., \& Shields, J. C. 2001, AJ, 121, 1648

Mattila, S., \& Meikle, W. P. S. 2001, MNRAS, 324, 325

Miknaitis, G., Pignata, G., Rest, A., et al. 2007, ApJ, 666, 674

Modjaz, M., Kewley, L., Kirshner, R. P., et al. 2008, AJ, 135, 1136

Moss, C., \& Whittle, M. 2005, MNRAS, 357, 1337

Moustakas, J., Kennicutt, Jr., R. C., \& Tremonti, C. A. 2006, ApJ, 642, 775

Ofek, E. O., Cameron, P. B., Kasliwal, M. M., et al. 2007, ApJ, 659, L13

Oke, J. B., \& Sandage, A. 1968, ApJ, 154, 21

Oke, J. B., \& Gunn, J. E. 1983, ApJ, 266, 713

Pastorello, A., Smartt, S. J., Mattila, S., et al. 2007, Nature, 447, 829

Patat, F., Cappellaro, E., Danziger, J., et al. 2001, ApJ, 555, 900

Perryman, M. A. C. 2005, in Astrometry in the Age of the Next Generation of

Large Telescopes, ed. P. K. Seidelmann, \& A. K. B. Monet, ASP Conf. Ser., 338,3

Pettini, M., \& Pagel, B. E. J. 2004, MNRAS, 348, L59

Pozzo, M., Meikle, W. P. S., Fassia, A., et al. 2004, MNRAS, 352, 457

Prieto, J. L., Stanek, K. Z., \& Beacom, J. F. 2008, ApJ, 673, 999

Pritchet, C. J., \& The SNLS Collaboration 2005, in Observing Dark Energy, ed. S. C. Wolff, \& T. R. Lauer, ASP Conf. Ser., 339, 60

Quimby, R. 2006, Central Bureau Electronic Telegrams, 644, 1

Quimby, R., Mondol, P., Hoeflich, P., et al. 2005a, Central Bureau Electronic Telegrams, 116, 1

Quimby, R. M., Castro, F., Gerardy, C. L., et al. 2005b, BAAS, 37, 1431

Quimby, R., Castro, F., Mondol, P., Caldwell, J., \& Terrazas, E. 2007a, Central Bureau Electronic Telegrams, 793, 1

Quimby, R. M., Aldering, G., Wheeler, J. C., et al. 2007b, ApJ, 668, L99
Richardson, D., Branch, D., Casebeer, D., et al. 2002, AJ, 123, 745

Riello, M., \& Patat, F. 2005, MNRAS, 362, 671

Sako, M., Bassett, B., Becker, A., et al. 2008, AJ, 135, 348

Salpeter, E. E. 1955, ApJ, 121, 161

Scannapieco, E. 2006, in press [arXiv: astro-ph/0609208]

Schiminovich, D., Ilbert, O., Arnouts, S., et al. 2005, ApJ, 619, L47

Schlegel, D. J., Finkbeiner, D. P., \& Davis, M. 1998, ApJ, 500, 525

Schmidt, B. P., Kirshner, R. P., Eastman, R. G., et al. 1994, ApJ, 432, 42

Smartt, S. J., Eldridge, J. J., Crockett, R. M., \& Mound, J. R. 2008, MNRAS, submitted

Smith, N., Chornock, R., Li, W., et al. 2008, ArXiv e-prints, 804

Smith, N., Li, W., Foley, R. J., et al. 2006, in press [arXiv: astro-ph/0612617]

Sollerman, J., Östlin, G., Fynbo, J. P. U., et al. 2005, New Astron., 11, 103

Stanek, K. Z., Gnedin, O. Y., Beacom, J. F., et al. 2006, Acta Astron., 56, 333

Stanek, K. Z., Matheson, T., Garnavich, P. M., et al. 2003, ApJ, 591, L17

Stasińska, G. 2005, A\&A, 434, 507

Takeuchi, T. T., Buat, V., \& Burgarella, D. 2005, A\&A, 440, L17

Tanvir, N. R., Barnard, V. E., Blain, A. W., et al. 2004, MNRAS, 352, 1073

Tchekhovskoy, A., McKinney, J. C., \& Narayan, R. 2008, ArXiv e-prints, 803

Tremonti, C. A., Heckman, T. M., Kauffmann, G., et al. 2004, ApJ, 613, 898

Vink, J. S., \& de Koter, A. 2005, A\&A, 442, 587

Woosley, S. E. 1993, ApJ, 405, 273

Woosley, S. E., \& MacFadyen, A. I. 1999, A\&AS, 138, 499

Woosley, S. E., \& Bloom, J. S. 2006, ARA\&A, 44, 507

Woosley, S. E., \& Heger, A. 2006, ApJ, 637, 914

Woosley, S. E., Blinnikov, S., \& Heger, A. 2007, Nature, 450, 390

Yoon, S., Langer, N., Cantiello, M., Woosley, S. E., \& Glatzmaier, G. A. 2008, ArXiv e-prints, 801

Yoon, S.-C., \& Langer, N. 2006, in Stellar Evolution at Low Metallicity: mass Loss, Explosions, Cosmology, ed. H. J. G. L. M. Lamers, N. Langer, T. Nugis, \& K. Annuk, ASP Conf. Ser., 353, 63

Yoon, S.-C., Langer, N., \& Norman, C. 2006, A\&A, 460, 199

Yuan, F., Akerlof, C., Quimby, R., et al. 2007, in American Astronomical Society Meeting Abstracts, 211, 105.05

Zeh, A., Klose, S., \& Hartmann, D. H. 2004, ApJ, 609, 952 